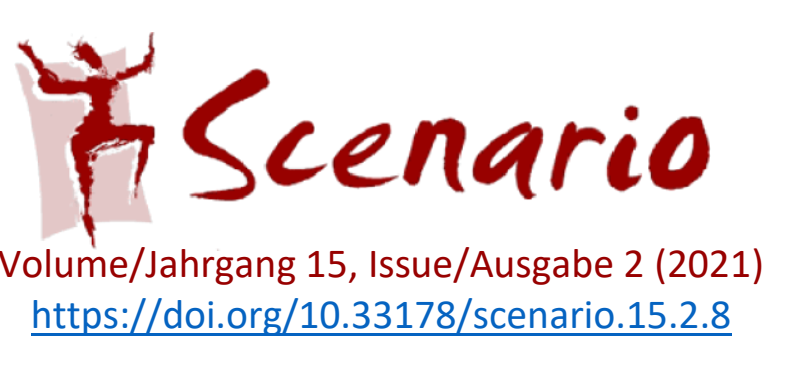

\title{
Englischlernende bearbeiten interkulturelle und dramapädagogische Sprechaktivitäten
}

\author{
Was passiert mit ihrer selbst wahrgenommenen Sprech- \\ kompetenz?
}

\section{Christiane Klempin \& Nicole Bosse}

Englisch als zweite Fremdsprache lernende Zehntklässler:innen eines Berliner Gymnasiums (n = 31) nahmen im Rahmen einer quasi-experimentellen Prä-Post-Interventionsstudie im Mixed Methods-Vertiefungsmodell an zwei fremdsprachlichen Lernangeboten teil: Ein erstes Lernangebot setzte auf Bearbeitung einer interkulturell kommunikativen Kompetenzaufgabe, ein zweites auf dramapädagogisches (d.h. performativ-ganzheitliches) Erleben der englischen Sprache. Beide Aktivitätenformate wurden den Zehntklässler:innen durch Englischlehramtsstudierende im Bachelor $(n=21)$ angeboten. Die interkulturellen wie performativen Aktivitäten sind im Zuge eines Theorie- und Praxiselemente verknüpfenden Seminarformates an der Freien Universität (FU) Berlin entstanden. Nachgegangen wurde der Frage, ob ein performatives im Vergleich zu einem interkulturellen Lernangebot positiver auf die selbst wahrgenommene Sprechkompetenz der Englischlernenden wirkt. Die qualitativen wie auch quantitativen Befunde dieser Studie verweisen auf einen positiven Einfluss performativer Lernangebote auf die Selbstwahrnehmung mündlicher Kompetenzen von Englischlernenden.

\section{Theoriehintergrund}

Der Wirkung Performativer Didaktik auf die Sprechkompetenz sind bisher bereits einige empirische Studien nachgegangen (u.a. Bora, 2020; Gill, 2013; Piazzoli, 2011; Sirisrimangkorn \& Suwanthep, 2013; Sirisrimangkorn, 2018;). Einige Studien konnten die positive Wirkung Performativer Didaktik auf die Sprechkompetenz nachweisen. In der Summe fallen die gesichteten empirischen Studien hinsichtlich Intensität und Länge der performativen Intervention (z.B. mehrere Wochen oder mehrere Monate) sowie Komplexität der angewandten Forschungsdesigns (z.B. mit oder ohne Kontrollgruppen) recht verschieden aus. Ihre Ergebnisse lassen sich daher nur schlecht miteinander vergleichen.

Sirisrimangkorn und Suwanthep (2013) verglichen über einen sechzehnwöchigen Interventionszeitraum hinweg zwei Englischstudierendengruppen ( $n=80$ ), die dieselben englischsprachigen Inhalte bearbeiteten. Die Experimentalgruppe (EG) nutzte performative Rollenspiele, während die Kontrollgruppe (KG) mit Lehrbuchaufgaben sowie darin 
Klempin \& Bosse: Englischlernende bearbeiten interkulturelle und dramapädagogische Sprechaktivitäten

enthaltenen Rollenspielen die Sprachinhalte bearbeitete. ${ }^{1}$ Zur Untersuchung stand die Sprechkompetenz der Englischstudierenden vor (prä) bzw. nach (post) der EG- oder KGIntervention. Erfasst wurde die Sprechkompetenz zum einen durch semistrukturierte Interviews und zum anderen durch Tagebücher der Studierenden. Insgesamt zeigte sich, dass die Sprechkompetenz der EG-Teilnehmenden im KG-Vergleich statistisch signifikant besser ausfiel. Galante und Thomson (2017) bestätigten in ihrer Studie jene positive Wirkung dramapädagogischer Aktivitäten mit abschließender Inszenierung ( $n_{E G}=13$ ) auf Englischlernende im Vergleich zu Unterricht ohne dramapädagogische Aktivitäten ( $n_{K G}=11$ ). Die Sprechfähigkeiten wurden vor und nach der Intervention anhand identischer Sprechaufgaben in beiden Gruppen kriteriengeleitet durch 30 Muttersprachler:innen erfasst. Positive Entwicklungen zeigten sich insbesondere hinsichtlich der Sprechflüssigkeit. Studien ohne installierte KG umfassten eine dramapädagogische Interventionsdauer von bis zu vier Monaten (Sirisrimangkorn, 2018) beziehungsweise anderthalb bis zu drei Monaten (Bora, 2020; Gill, 2013; Piazzoli, 2011). Anhand von Videoaufnahmen der dramapädagogischen Workshops, eines Reflexionstagebuchs der Forschenden, semistrukturierter Interviews mit den Studierenden, nicht weiter beschriebenen Concept Mapping-Diagrammen und einem Stimulated Recall-Interview zu drei videografierten Abschnitten konnte beispielsweise Piazzoli (2011, S. 569) zeigen, dass Process Drama ${ }^{2}$ positiv auf die Sprechflüssigkeit wirkt. Sirisrimangkorn (2018) untersuchte die Prä-Post-Sprechkompetenzentwicklung von 23 Schüler:innen, wobei ebenfalls eine positive Entwicklung ausgemacht werden konnte. Keine Zuwächse zeigten sich für die Untersuchungsteilnehmenden nach zwei performativen Einzelinterventionen in einer Studie von Gill (2013). Die Studie umfasste zehn Studierende, deren Sprechkompetenz anhand eines Prä-Post-Selbsteinschätzungsbogens und Urteilen der Forschenden bestimmt wurde. ${ }^{3}$ Bora (2020) unterrichtete zehn Schüler:innen zehn Wochen lang nach einem performativen (EG) und einem teils performativen Ansatz (KG), konnte aber keine (selbst wahrgenommenen) Sprechkompetenzzuwächse der Lernenden auflösen. Dennoch entdeckte Bora leichte Unterschiede hinsichtlich einer positiveren Wahrnehmung des performativen Unterrichtsansatzes, vor allem hinsichtlich der Verfügbarkeit sprachlicher Mittel und der Sprechflüssigkeit der Lernenden. Die Erhebungsgrundlage bildeten ein Selbsteinschätzungsfragebogen und Einzelinterviews nach jeder Intervention.

\footnotetext{
1 Sirisrimangkorn und Suwanthep (2013) machen keine Aussagen über die Stichprobenzusammensetzung. Außerdem führten die Studierenden auch in der KG dramapädagogische Rollenspiele zur Spracharbeit aus, weshalb zweifelhaft ist, ob es sich tatsächlich um eine angemessene Vergleichsgruppe für die EG handelte.

2 Process drama beschreibt eine Lehrmethode, bei welcher auf Grundlage von beispielsweise Texten „(performative) Unterrichtsaktivitäten“ (Schewe, 2017, S. 50) geschaffen werden, mit dem Ziel der Herstellung "eines fiktiven Rahmens" (Schewe, 2017, S. 50) und der Weiterentwicklung der dramatischen Handlung.

${ }^{3}$ Die berichteten Positivwirkungen nach allen Teilinterventionen können jedoch nicht auf die Dramaaktivitäten zurückgeführt werden, da auch nicht-performative Elemente Interventionsbestandteil waren und es an einer Kontrollgruppe fehlte.
} 
Klempin \& Bosse: Englischlernende bearbeiten interkulturelle und dramapädagogische Sprechaktivitäten

Insbesondere Studien mit längeren mehrmonatigen Interventionen gingen insgesamt mit positiven Sprechkompetenzentwicklungen einher. Piazzoli (2011) sowie Galante und Thomson (2017) konnten so etwa erste empirische Nachweise für Zuwächse der Sprechflüssigkeit erbringen. Auffällig bei den genannten Studien sind häufig der Verzicht auf die Installation von Vergleichsgruppen (ohne oder mit einer alternativen performativen Behandlung), kleinere Stichproben sowie fehlende Randomisierungen der Proband:innen. Zusammen betrachtet führt dies dazu, dass die Befunde der betrachteten Studien mit Zurückhaltung interpretiert werden sollten und nur eingeschränkt auf andere Kontexte performativen Lehrens und Lernens übertragen werden können. Außerdem befördert dies die Notwendigkeit weiterer Untersuchungen zum Zusammenhang zwischen dramapädagogisch gestütztem Fremdsprachenerwerb, in der Fachwelt zunehmend auch unter dem Begriff der Performativen Didaktik (PD) ${ }^{4}$ gefasst, und der Förderung fremdsprachlicher Erwerbsprozesse. Das Drama Lab an der FU Berlin (Klempin, 2019; Klempin, 2020) soll daher den Ausgangspunkt einer empirischen Quasi-Experimentalstudie bilden, um einen weiteren Beitrag zur datengestützten Fundierung performativer Didaktik und deren Nutzen für den Fremdsprachenerwerb leisten zu können.

\section{Interventionsbeschreibung}

Das Drama Lab im Format eines sog. Lehr-Lern-Labor-Seminars im Fach Englisch (Klempin, 2019) $)^{5}$ bildet den Kontext, in dem diese Mixed Methods-Studie durchgeführt wurde. Das Drama Lab (Klempin, 2020) ${ }^{6}$ fand in einem Seminarraum der FU Berlin statt und ermöglicht Lehramtsstudierenden eine theoriebasierte Planung von (Englisch)-Unterricht, dessen Durchführung unter komplexitätsreduzierten Bedingungen (Rehfeldt et al., 2019) sowie eine anschließende systematische Reflexion erster Praxiserfahrungen. Die Studierenden eigneten sich das theoretische Wissen unter Anleitung der Dozierenden mithilfe ausgewählter Fachliteratur und durch handlungsorientierte Selbsterkundung interkultureller

\footnotetext{
${ }^{4}$ Performative Fremdsprachendidaktik kann verstanden werden als „stark an den Künsten orientiert und sich nicht nur als Wissenschaft, sondern ebenso als Kunst begreif[end] “ (Schewe, 2015, S. 31, zitiert nach Miladinović, 2019, S. 11). Miladinović (2019, S. 11-12) argumentiert, dass performative Fremdsprachendidaktik als Schirmbegriff verwendet wird, um „alle Formen der Fremdsprachenvermittlung zu kennzeichnen, die sich aus den Künsten bzw. den mit diesen assoziierten (kulturspezifischen) Formen drama-/theaterpädagogischer Praxis ableiten lassen“ (Schewe, 2015, S. 33, zitiert nach Miladinović, 2019, 11-12).

5 Das diesem Artikel zugrundeliegende Vorhaben wird im Rahmen der gemeinsamen „Qualitätsoffensive Lehrerbildung“ von Bund und Ländern mit Mitteln des Bundesministeriums für Bildung und Forschung unter dem Förderkennzeichen 01JA1502 (Projekt K2teach, FU Berlin) gefördert. Die Verantwortung für den Inhalt dieser Veröffentlichung liegt bei den Autorinnen.

${ }^{6}$ Das Drama Lab ist im Rahmen einer Handreichung (zu Kompetenzzielen, zur Sitzungsgestaltung, zu Methoden und Sozialformen, etc.) ausführlich beschrieben worden: https://tinyurl.com/w4zvrnsx (Zugriff am: 09.04.2021). Ein Bericht zum Drama Lab gibt weitere Einblicke, v.a. auch bezüglich der Wahrnehmung des Drama Labs durch die Teilnehmenden: https://www.fu-berlin.de/sites/k2teach/im-gespraech/EinblickLehre/DramaLab/index.html (Zugriff: 09.04.2021).
} 
Klempin \& Bosse: Englischlernende bearbeiten interkulturelle und dramapädagogische Sprechaktivitäten

beziehungsweise performativer Aktivitäten an. Als anschließende Planungs- und Reflexionsgrundlage selbst erlebter Unterrichtspraxis diente ein Raster zu folgenden Aspekten: Bezug der Aktivität zum (Berliner) Rahmenlehrplan, begründete, kleinschrittige Vorgehensweise, mögliche Lernhindernisse, Zeitmanagement und Sozialform. Weiterhin unterstützten sich die Studierenden bei der Planung und Reflexion durch gegenseitiges Feedback. ${ }^{7}$ Für das Drama Lab durchlaufen Teilnehmende den nachfolgenden 7-schrittigen Zyklus:

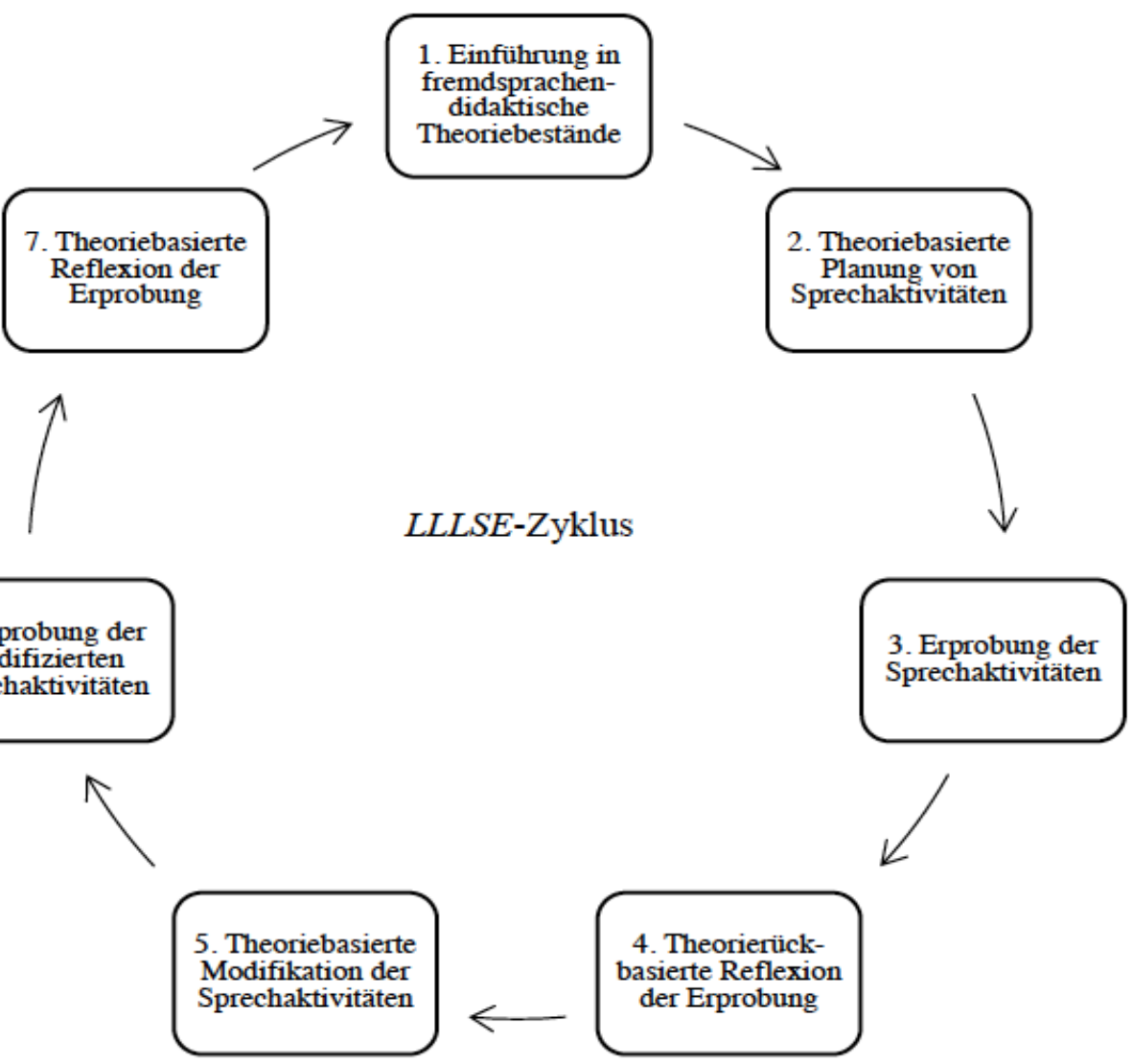

Abbildung 1: Zyklischer Ablauf des Drama Labs als Lehr-Lern-Labor-Seminar im Fach Englisch (LLLSE) in fünf Schritten (Klempin, 2019, S. 141)

In diesem Quasi-Experiment zum Drama Lab wurden die Theoriephasen (vgl. Abbildung 1, Phasen 1-2 und 4-5) durch zwei neue Theoriebausteine ersetzt: Interkulturelle Kommunikative Kompetenz (IKK) und Performative Didaktik (PD). In den ersten Wochen setzten sich die Bachelorlehramtsstudierenden zunächst mit IKK-Theoriebeständen auseinander (u.a. Byram, 1997; Grau \& Würffel, 2003) und planten darauf aufbauend 30-minütige IKK-Lernangebote für eine Klasse von Zehntklässler:innen, mit denen sie diese Aktivitäten daraufhin erprobten.

\footnotetext{
${ }^{7}$ In einer ersten Studie im quasi-experimentellen Mixed-Methods-Design (2015-2018) in der Englischdidaktik an der FU Berlin erwies sich das Lehr-Lern-Labor-Seminar-Konzept als sehr wirksam in Bezug auf die Reflexionskompetenzsteigerung der Studierenden in Tiefe sowie Breite, auch im Kontrast zu einem klassischen didaktischen Theorieseminar und einem weiteren Lehr-Lern-Labor-Seminar der Englischdidaktik (vgl. Klempin, 2019).
} 
Klempin \& Bosse: Englischlernende bearbeiten interkulturelle und dramapädagogische Sprechaktivitäten

Dabei sollten die Lernenden auf Grundlage von Lernmaterialien (z.B. Texte, Medien, usw.) zur Auseinandersetzung und Reflexion mit anderen Sichtweisen angeregt werden. Im Anschluss folgten eine Reflexionssitzung sowie der zweite Theoriebaustein zu handlungsorientiertem Fremdsprachenunterricht inklusive der Beschäftigung mit den PD-Prinzipien (hierzu Theorieund Praxislektüren im Drama Lab u.a. von Baldwin, 2004; Piccardo, 2014). Unter den von den Studierenden entwickelten Lernangeboten fanden sich insgesamt 18 Rollenspiele, vier Pantomime-Aktivitäten und eine Hot Seat-Aktivität.

Während in der ersten Studie zur Wirksamkeit eines Lehr-Lern-Labor-Seminars im Fach Englisch die Förderung und Messung der studentischen Reflexionskompetenzen im Zentrum stand (vgl. Klempin, 2019), soll sich in dieser zweiten Studie nun den Wirkungen der studentischen Aktivitätenangebote (PD und IKK) auf die Lernenden zugewendet werden. Dies ist der Erkenntnis geschuldet, dass insbesondere für geisteswissenschaftliche Lehr-LernLabore (LLL) bisher weitestgehend unbekannt ist, wie dieses Format auf (Fremdsprachen)Lernende wirkt (Rehfeldt et al., 2018). Daher soll mit dieser Studie erstmalig für die Englischdidaktik der Frage nachgegangen werden, ob und inwieweit von interkulturellen bzw. performativen Lehr-Lernangeboten für Englischlernende sprechförderliche Wirkungen ausgehen (s. quantitativ: Hypothesen 1-2 (H1-H2), qualitativ: Forschungsfragen 1-4 (FF1FF4)): ${ }^{8}$

H1: Die Englischlernenden schätzen ihre Sprechkompetenzen bei PD- anders ein als bei IKKLernangeboten.

H2: Die Englischlernenden schätzen sprechmotivationale Aspekte (Spaß, Interesse, Einfluss der Peers, Aufwärmeffekt, Fehlerkultur, Unterstützung, abweichende Lehrperson) bei PDanders ein als bei IKK-Lernangeboten.

FF1: Wie schätzen Englischlehramtsstudierende den Mehrwert von IKK- und PDLernangeboten ein?

FF2: Welche Hürden nehmen die Englischlehramtsstudierenden für IKK- und PD-Lernprozesse wahr?

FF3: Wie schätzen die Englischlehramtsstudierenden die Lernatmosphäre während der IKKund PD-Lernangebote ein?

\footnotetext{
${ }^{8}$ Für die qualitative Teilstudie wurde auf die Formulierung gerichteter Forschungshypothesen verzichtet. Dies entspräche schlichtweg nicht den der dokumentarischen Methode (Bohnsack, 2014) zugrundeliegenden forschungsmethodischen Grundprinzipien, d.h. Offenheit des Forschenden gegenüber dem Forschungsgegenstand, gegenüber der Interpretation der Daten sowie dem Ausgang der Analysen. Es wurden daher offenere Forschungsfragen formuliert (FF1-FF4).
} 
Klempin \& Bosse: Englischlernende bearbeiten interkulturelle und dramapädagogische Sprechaktivitäten

\section{Erhebungsmodalitäten}

Die Datenerhebungen erfolgten unter der Vorannahme des sog. Solomon-Vier-GruppenVersuchsplans (Rost, 2013, S. 145f; Solomon, 1949). Der Solomon-Vier-Gruppen-Plan zeichnet sich durch vier Wiederholungen aus und bietet den Vorteil, dass EG auch als KG fungieren und vice versa. Dieser Studientyp liefert zuverlässige Daten, zählt aber auch zu den komplexesten experimentellen Versuchsplänen. Es findet demnach selten Anwendung in der LehrLernforschung (Klempin \& Wirag, 2020; Rost, 2013). Die quantitativen Datenerhebungen fanden zu zwei Messzeitpunkten (MZP), prä (Test 1) und post (Test 2), jeweils im Anschluss an die IKK- bzw. PD-Aktivitäten statt. Gemäß Solomon-Design werden in diesem Bericht ausschließlich die Ergebnisse des ersten Durchlaufs des Gesamtversuchsplans berichtet (s. Drama Lab 1, fett markiert):

1. Drama Lab 1: IKK - Test 1 - PD - Test 2

2. Drama Lab 2: PD - Test 1 - IKK - Test 2

3. Drama Lab 3: $\mathrm{CLT}^{9}-$ Test $1-\mathrm{PD}-$ Test 2

4. Drama Lab 4: PD - Test 1 - CLT - Test 2

Die qualitative Datenerhebung bestand aus einer Post-Erhebung in Form von zwei Gruppendiskussionen in der vorletzten Drama Lab-Sitzung. Dieses Vorgehen entspricht der Logik eines Vertiefungsmodells (Mayring, 2001, S. 5), bei dem zunächst quantitatives Datenmaterial erhoben und ausgewertet und anschließend qualitative Analysen angeschlossen werden, um etwaige Erkenntnislücken der einen oder anderen Forschungslogik füllen zu können. Dadurch soll gewährleistet werden, dass die quantitativ gewonnenen Schüler:innendaten mit den qualitativ gewonnenen Studierendenurteilen bzgl. der wahrgenommenen Interaktionsqualität zu einem späteren Zeitpunkt abgeglichen werden können. Eine solche methodische Triangulation ist sinnvoll, um den Einschränkungen zu begegnen, die bei quasi-experimentellen Studien üblicherweise zu verzeichnen sind.

\subsection{Quantitative Datenerhebungen}

An der Studie nahm eine 10. Klasse eines Berliner Gymnasiums mit 31 Schüler:innen teil, davon 16 Mädchen und 15 Jungen. 27 Schüler:innen waren zur zweiten Intervention anwesend, davon 13 Mädchen und 14 Jungen. Nach der Erstintervention IKK lag der

\footnotetext{
9 In einer dritten Iteration wird der IKK-Theoriebaustein durch die Theorie des Communicative Language Teaching (CLT) ausgetauscht, um zu einem späteren Zeitpunkt den statistischen Einfluss dieses fremdsprachendidaktischen Ansatzes aus dem von PD herausrechnen und den wahren Einfluss von PD erfassen zu können.
} 
Klempin \& Bosse: Englischlernende bearbeiten interkulturelle und dramapädagogische Sprechaktivitäten

Fragebogenrücklauf bei 30 Schüler:innen, nach der Zweitintervention PD bei 21. Nach Aussage der Englischlehrenden hatten die Lernenden zu Interventionsbeginn wenig bis sehr wenig Erfahrung mit Dramaaktivitäten. Die Lernenden nahmen Auswahl und Reihenfolge der studentischen Lehr-Lernangebote im Drama Lab selbst vor, sodass von einer gewissen Selbstläufigkeit und damit auch Zufälligkeit (Stichwort: Randomisierung) ausgegangen werden kann.

Während ihrer beiden Besuche an der FU Berlin - zur Erinnerung: der erste Besuch diente den IKK- und der zweite den PD-Lernangeboten - konnten die Englischlernenden jeweils drei bis maximal vier 30-minütige Lernangebote wahrnehmen (vgl. Klempin, 2020, S. 12). Nach Abschluss der IKK- bzw. PD-Aktivitäten wurde zu beiden Besuchsterminen ein Fragebogen ${ }^{10}$ ausgegeben, mit dem die Schüler:innen ihre momentane mündliche Sprechkompetenz selbst einschätzen konnten. ${ }^{11}$

Als Vorlage für die Entwicklung eines Fragebogens zur Selbsteinschätzung der wahrgenommenen Sprechkompetenzen diente das Beurteilungsraster mit validierten Deskriptoren A1-C2 zur mündlichen Kommunikation des Gemeinsamen Europäischen Referenzrahmens (GeR; Trim, North \& Coste, 2013, S. 37-38). Um Verständnisschwierigkeiten des Rasters aufzudecken, wurden jeweils zwei kognitive Interviews gemäß des „lauten Denkens" (Bethmann, Buschle \& Reiter, 2019, S. 167) durchgeführt. Interviewpartner:innen waren zwei Zehntklässler:innen eines Berliner Gymnasiums, welche jeweils vor der Entwicklung eines Fragebogenentwurfs und nach dessen Erstellung interviewt wurden. ${ }^{12}$ Die Schüler:innen wurden im Zuge dessen gebeten, ihre Sprechkompetenz für fünf Kategorien des Rasters anhand der Deskriptoren A1-C2 laut verbalisierend einzuschätzen (Trim, North \& Coste, 2013, S. 37): 1. Wortschatzgröße und Satzkomplexität, 2. Korrektheit, 3. Flüssigkeit, 4. Fähigkeit zur Aufrechterhaltung einer Konversationsinteraktion und 5. Kohärenz. Analog zum Ausgangsraster ergibt sich für diese Studie demgemäß ein fünf Items umfassender Fragebogen. Die Niveaustufen A1-C2 werden durch eine sechsstufige Likertskalierung dieser fünf Items abgebildet: 1. Wortschatzgröße und Satzkomplexität, 2. Grammatische Korrektheit, 3. Flüssigkeit, 4. Konversationsfähigkeiten und 5. Zusammenhängendes Sprechen. Um der Forderung Schmenks (2015, S. 42) nachzukommen, den affektiven und subjektiven Mehrwert von PD im Vergleich zu anderen Lehransätzen zu untersuchen, wurden auch motivationale Einflussvariablen wie beispielsweise der Spaß der Lernenden während der Aktivitäten sowie

\footnotetext{
${ }^{10}$ Der Fragebogen kann hier eingesehen werden.

11 Die Schüler:innen wurden darum gebeten, ihre selbst wahrgenommene Sprechkompetenz hinsichtlich des/derjenigen Studierenden zu beurteilen, bei dem/der sie zuletzt eine Aktivität durchgeführt hatten. Für das Ausfüllen des Fragebogens benötigten die Schüler:innen ungefähr fünf Minuten.

${ }^{12}$ Aufgrund dessen, dass sich das GeR-Beurteilungsraster hauptsächlich an Lehrende richtet und daher sprachlich sehr komplex ausfällt, mussten am Instrument leichte sprachliche Modifikationen vorgenommen werden.
} 
Klempin \& Bosse: Englischlernende bearbeiten interkulturelle und dramapädagogische Sprechaktivitäten

deren Interesse an den Angebotsinhalten als Fragebogenitems aufgenommen. Außerdem sollte geprüft werden, ob und inwieweit vorhergehende von den Zehntklässler:innen wahrgenommene Lernangebote des Drama Labs, eine fehlerfreundliche Lernkultur sowie die Unterstützung der Lernprozesse durch die Lehramtsstudierenden (z.B. in Form von Scaffolding) Einfluss auf die Sprechkompetenz hatten. Zwei Items zur wahrgenommenen Lernatmosphäre erfassten mögliche Einflüsse durch die Mitschüler:innen (o.a. Peers) oder die Lehramtsstudierenden als Lehrpersonen, die den Schüler:innen zunächst unvertraut waren. Die Sichtung der studentischen IKK- und PD-Planungsentwürfe sollte zudem der Identifikation performativer Anteile in den IKK-Lernangeboten dienen. Denn aufgrund der konzeptuellen Nähe von IKK und PD waren Überlappungen wahrscheinlich (u.a. Bosenius, 2017, S. 53). Ein geringer Anteil von PD-Elementen in den IKK-Aktivitäten könnte darauf hindeuten, dass die Lehr-Lernansätze konzeptuell verschieden sind und demnach zwei getrennte Interventionen darstellen. Jedoch ist nicht auszuschließen, dass der geringere Fokus auf PD-Elementen im IKKTeil des Seminars auch zu einer geringeren Integrierung eben dieser Elemente führt.

\subsection{Qualitative Datenerhebungen}

In der vorletzten Sitzung des Drama Labs (vgl. Abbildung 1, Schritt 7), als Teil der zweiten Reflexionssitzung, fanden zwei Gruppendiskussionen statt. Dazu wurden die Studierenden mit der Split-Half-Methode zufällig zwei Gruppen zugeordnet: 1. einer fortan als PD-Gruppe deklarierten und 2. einer IKK-Gruppe. Das Interview mit der PD-Gruppe wurde durch die Seminarleiterin und Erstverfasserin dieses Berichtes geführt und das mit der IKK-Gruppe durch eine studentische Mitarbeiterin. Ein zuvor entwickelter Leitfaden ${ }^{13}$, der Frageimpulse und Dauer (45 Minuten) vorgab, stellte sicher, dass beide Gruppendiskussionen analog geführt wurden. Die Fragen des Leitfadens unterschieden sich für beide Gruppen ausschließlich in den Theoriebezügen: Für die IKK-Gruppe lag der Schwerpunkt auf den IKK- und für die PD-Gruppe auf den PD-Theoriebeständen (s. Fragenblock B: entweder PD- oder IKK-Theorie). ${ }^{14}$ Fragenblock A war von dieser Differenzierung nicht betroffen, da die Studierenden lediglich ihren Gesamteindruck bezüglich des Drama Labs berichten konnten. Der Fragenimpuls lautete hierfür:

Wie habt ihr das Seminar "Grundlagen der Fachdidaktik Englisch: Lehr-Lern-Labor" grundsätzlich empfunden? Erzählt doch mal.

\footnotetext{
${ }^{13}$ Der Leitfaden kann hier eingesehen werden.

${ }^{14}$ Ein solches analoges Erhebungsvorgehen in beiden Gruppen ist, strenggenommen, nicht im Sinne der Gruppendiskussion als zentralem Erhebungsformat der dokumentarischen Methode. Da diese Studie jedoch auf die Verknüpfung quantitativer und qualitativer Daten und Befunde abzielt, ist es an dieser Stelle von Bedeutung gewesen, zwei komplementäre Erhebungssettings herzustellen.
} 
Klempin \& Bosse: Englischlernende bearbeiten interkulturelle und dramapädagogische Sprechaktivitäten

Während die PD-Gruppe (Interviewlänge: 41:39 Minuten, 9 Teilnehmer:innen: $m=1, w=8$ ) im Seminarraum für die Gruppendiskussion zusammenkam, wurde die IKK-Gruppe auf dem Flur in einer Sitzgruppe interviewt (Interviewlänge: 36:17 Minuten, 12 Teilnehmer:innen: $m$ = $1, \mathrm{w}=11) .{ }^{15}$

\section{Datenauswertung}

Die Datenauswertung für die quantitativen und qualitativen Daten erfolgte getrennt voneinander, sodass eine Datentriangulation, die tatsächliche Zusammenführung der beiden Datentypen, erst auf der Ebene der Ergebnisinterpretation stattfand. Die quantitativen Daten ermöglichen den Nachvollzug der Entwicklung der abhängigen Variable (AV: selbst beurteilte Sprechkompetenzentwicklung der Schüler:innen) unter Einfluss der beiden unabhängigen Variablen (UV1: IKK-Lernangebot; UV2: PD-Lernangebot).

\subsection{Quantitative Datenauswertung}

Zunächst wurden die Verteilungen für alle Fragebogenitems auf Kurtosis und Schiefe geprüft. Anschließend wurden die Werte der ersten fünf Items (1. Wortschatzgröße und Satzkomplexität, 2. Grammatische Korrektheit, 3. Flüssigkeit, 4. Konversationsfähigkeiten und 5. Zusammenhängendes Sprechen) pro Schüler*in gemittelt. Aus allen gemittelten Werten wurde dann der Median der selbst wahrgenommenen Sprechkompetenz der Schüler:innen für UV1 und UV2 berechnet. ${ }^{16}$ Diese Kennwerte dienten einer ersten Identifizierung von Unterschieden zwischen UV1 und UV2. Für die anschließende Berechnung signifikanter Unterschiede zwischen den Interventionen boten sich der t-test (bei normalverteilten) oder der Mann-Whitney-U-Test (bei nichtnormalverteilten Daten) an (Falk et al., 2014, S. 150; Wollschläger, 2017, S. 237).

1. Da Varianzhomogenität (Gleichheit der Varianzen) der zu vergleichenden Daten eine Voraussetzung für die Durchführung des t-tests bei normalverteilten Daten ist, wurde zunächst der Levene-Test angewendet (Wollschläger, 2017, S. 231ff.).

a. Bei Varianzheterogenität (Ungleichheit der Varianzen) der normalverteilten Daten wurde nicht der t-test, sondern der Welch-Test mittels des Statistikprogramms $R$ (Version 3.6.3) mit einem Signifikanzniveau von $p<.05$ durchgeführt.

b. Für den Mann-Whitney-U-Test bei nicht normalverteilten Daten wurde aufgrund der vorliegenden Stichprobe mit mehr als 20 Teilnehmenden ( $n 1>20$

\footnotetext{
${ }^{15}$ Diese Faktoren werden in den Studienlimitationen nochmals aufgegriffen.

16 Der Median wurde hier anstelle des gängigen Mittelwerts gewählt, da die Itemmehrzahl keine Normalverteilung aufwies. Der Einfluss von Ausreißern auf das Ergebnis konnte somit reduziert werden.
} 
Klempin \& Bosse: Englischlernende bearbeiten interkulturelle und dramapädagogische Sprechaktivitäten

und n2 > 20) sowie aufgrund verbundener Ränge die Normalverteilung approximiert (Falk et al., 2014, S. 155f.). ${ }^{17}$

2. Die Effektstärken wurden mit folgenden Maßen berechnet:

a. Pearsons $r$ bei nicht normalverteilten Daten,

b. Cohens $d$ bei normalverteilten Daten mit ihren jeweiligen Konfidenzintervallen (KI), Konfidenzniveau 95\%.

Da Effektstärken nicht mehr von der Stichprobengröße beeinflusst werden (anders als ein Signifikanztest), kann das Effektstärkenmaß zu einer realistischeren Einschätzung möglicher interventionsbedingter Unterschiede beitragen.

\subsection{Qualitative Datenauswertung}

Nachfolgend wird erläutert, wie die beiden Gruppendiskussionen - eine zu IKK- und eine zu PD-Theorien befragte Gruppe im Drama Lab - entlang dieser drei Leitlinien ausgewertet wurden:

- Hinsichtlich des eingeschätzten Mehrwerts von IKK und PD-Lernangeboten, durch den auf die Überzeugungen der Lehramtsstudierenden geschlossen werden kann

- Hinsichtlich der wahrgenommenen Hürden im IKK- und PD-Lernprozess

- Hinsichtlich der eingeschätzten Lernatmosphäre während der IKK- und PDLernangebote

Für die Datenauswertung wurden zunächst beide Gruppendiskussionen transkribiert. Daraufhin schloss die Auswertung mittels dokumentarischer Methode an, angelehnt an Bohnsack (2014), und durchgeführt in drei Hauptschritten durch drei Auswertende (Klempin und zwei studentische Mitarbeiter:innen mit Erfahrungen in qualitativer Forschung und Grundkenntnissen in der Englischdidaktik): ${ }^{18}$

1. Zunächst wurden die studentischen Aussagen durch kurze Paraphrasen der aufgegriffenen Themen und Inhalte entsprechend „formulierende[r] Interpretation“ nach Bohnsack (2014, S. 136) beschrieben.

2. Anschließend wurden die Diskursbewegungen der studentischen Aussagen entsprechend ihrer Gruppenzugehörigkeit und gemäß „reflektierende[r]

\footnotetext{
${ }^{17}$ Aufgrund der Stichprobengröße und der verbundenen Ränge (mehr als ein Stichprobenwert tritt öfter auf) wird die Streuung der Ränge verringert, sodass die Normalverteilung approximiert werden kann.

${ }^{18}$ Die Schritte eins und zwei wurden vorrangig durch studentische Mitarbeiter:innen geleistet. Der finale dritte Schritt der komparativen Analyse erfolgte begleitend zu den ersten beiden Schritten durch die Erstautorin.
} 
Klempin \& Bosse: Englischlernende bearbeiten interkulturelle und dramapädagogische Sprechaktivitäten

Interpretation" nach Bohnsack (2014, S. 137) durch zwei Analysierende erfasst (Publikation in Vorbereitung).

3. In einem finalen Schritt wurden fallimmanente und -exmanente Vergleiche für die Gruppen entsprechend Typenbildung nach Bohnsack (2014) angestellt (Publikation in Vorbereitung).

In diesem Beitrag geht es ausschließlich um die von den Befragten benannten Inhalte und Themen als sog. „kommunikativ[es] Wissen” (Bohnsack, 2014, S. 64), die sich im Zuge der formulierenden Interpretation (s. Schritt 1) aus dem Datenmaterial herauslösen ließen. In Folgebeiträgen wird ausführlich über die Befunde der "Analyse[schritte zwei und drei] von Sinnstrukturen jenseits des wörtlichen oder referenziellen Sinngehalts, aber auch jenseits der kommunikativen Absichten der Beteiligten" (Bohnsack, 2014, S. 378) berichtet. ${ }^{19}$

\section{Ergebnisse}

Im Folgenden werden zunächst die Ergebnisse des quantitativen Studienstrangs entlang der fünf Forschungshypothesen ( $\mathrm{H} 1-\mathrm{H} 5)$ berichtet und entlang der Forschungstheorie diskutiert (vgl. Kapitel 1). Auf quantitative folgen qualitative Studienergebnisse, die das Verständnis der selbst eingeschätzten Sprechkompetenz der Englischlernenden ergänzen und die die für Quasi-Experimente gängigen Studienbeschränkungen relativieren sollen.

\subsection{Quantitative Ergebnisse}

H1: Die Englischlernenden schätzen ihre Sprechkompetenzen bei PD-anders ein als bei IKKLernangeboten.

Ein erster Vergleich der Mediane mündlicher Kommunikation lässt einen Unterschied in der Selbsteinschätzung zwischen beiden Interventionen, IKK und PD, erkennen:

\footnotetext{
${ }^{19}$ Der vollständige Bericht bezieht sich auf alle Fragenblöcke der Gruppendiskussionen und spezifiziert daneben auch den Auswertungsprozess, der in diesem Beitrag der Knappheit wegen nur kurz umrissen werden kann.
} 
Klempin \& Bosse: Englischlernende bearbeiten interkulturelle und dramapädagogische Sprechaktivitäten

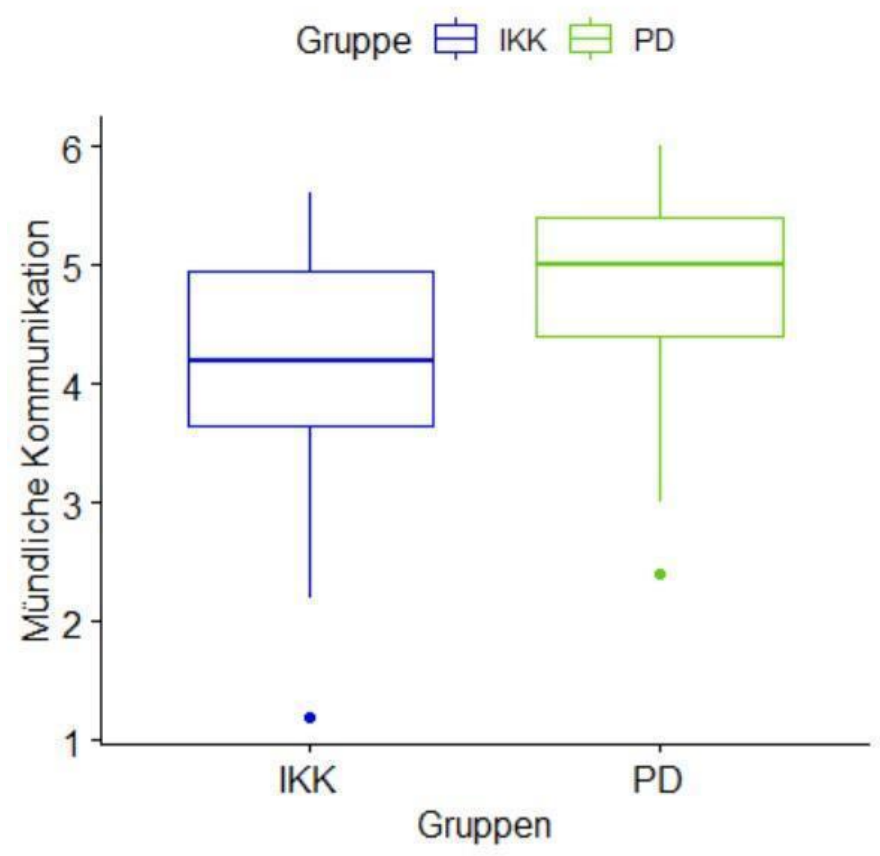

Abbildung 2: Selbsteinschätzung mündlicher Kommunikation bei den Interventionen IKK und PD

Die Schüler:innen schätzten ihre Sprechkompetenz nach den PD-Lernangeboten signifikant besser ein $(M e d=5)$ als nach den IKK-Lernangeboten (Med $=4.2), p=.01$ (s. Abb. 2). Die Effektstärke entsprechend Pearson liegt bei $r=0.33$ (KI: 0.04-0.52). Insbesondere die folgenden Items bestechen durch signifikante Unterschiede:

- Wortschatzgröße und Satzkomplexität, $p=.008, d=0.78$ (KI: 0.19-1.37),

- Flüssigkeit, $p=.03, d=0.64$ (KI: 0.56-1.23),

- Konversationsfähigkeiten, $p=.01, r=0.31$ (KI: 0.02-0.51) und

- Zusammenhängendes Sprechen, $p=.04, r=0.26$ (KI: -0.01-0.47).

Indes konnte kein signifikanter Unterschied für die Selbsteinschätzung der Grammatischen Korrektheit beobachtet werden, $p=.33, r=0.17$ (KI: -0,12 - 0.4). Die Effektstärken verweisen mit Ausnahme von Zusammenhängendem Sprechen und Grammatischer Korrektheit auf einen mittelstarken Effekt. Trotz des Ausbleibens eines signifikanten Unterschieds für Grammatische Korrektheit konnte eine kleine Effektstärke identifiziert werden, wobei sich die Schüler:innen nach der PD-Intervention (Med $P D=5$ ) etwas besser einschätzten als nach der IKK-Intervention (Med ${ }_{I K K}=4.5$ ). Zusammenhängendes Sprechen erzielte für beide Lernangebote identische Werte (Med IKK / $P D=5$ ). Gleichzeitig konnte aber eine mittlere Effektstärke nachgewiesen werden $(r=0.26)$. Da sich die Schüler:innen hinsichtlich ihrer mündlichen Kommunikation über alle Items hinweg bei der PD- besser als bei der IKKIntervention beurteilten, wurde nachträglich ein einseitiger Mann-Whitney-U-Test durchgeführt mit der Alternativhypothese H1: Die Selbsteinschätzung Zusammenhängenden 
Klempin \& Bosse: Englischlernende bearbeiten interkulturelle und dramapädagogische Sprechaktivitäten

Sprechens fällt bei PD besser aus als bei IKK. ${ }^{20}$ Die Testergebnisse unterstützen diese Hypothese, denn hinsichtlich Zusammenhängenden Sprechens beurteilten die Schüler:innen sich nach den PD-Lernangeboten besser als nach den IKK-Lernangeboten, $p=.02$.

H2: Die Englischlernenden schätzen sprechmotivationale Aspekte bei PD-Lernangeboten anders ein als bei IKK-Lernangeboten.

Die Schüler:innen nahmen den Faktor Spaß bei den PD-Aktivitäten stärker wahr $\left(\right.$ Med $\left._{P D}=6\right)$ als bei den IKK-Angeboten (Med ${ }_{I K K}$ ), $p=.0005, r=0.45$ (KI: $0.28-0.58$ ). Ein ähnliches Bild ergibt sich für den Einflussfaktor Interesse (Med ${ }_{P D}=5.5 \mathrm{im}$ Vergleich zu Med ${ }_{I K K}=4$ ), $p=.004$, $r=0.37$. Vergleicht man beide sprachlichen Lernangebote IKK und PD, dann entspricht das einem mittelstarken Effekt ( $r=0.37$ ). Die Peers scheinen währenddessen keine messbare Wirkung auf die wahrgenommene Sprechmotivation beider Lernangebote gehabt zu haben. Zeitgleich ergibt sich mit, wenn auch kleinem Effekt, $r=0.23$ (KI: $-0.04-0.41)$, zugunsten der PD-Lernangebote ein wahrgenommener positiver Einfluss der Lernatmosphäre auf die Sprechmotivation (Med ${ }_{P D}=6 \mathrm{im}$ Vergleich zum Med ${ }_{I K K}=5$ ). Keine statistisch signifikanten Veränderungsmaße in Abhängigkeit vom jeweiligen Lernangebot existieren derweil für den Einfluss vorhergehender Lernangebote, als sog. Aufwärmeffekt, oder für die Gewöhnung an eine Lehr-Lernmethode (Med $P D+I K K=5), p=.78$. Daneben scheinen die Fehlerkultur des Lehramtsstudierenden (Med $P D+I K K=6), p=.397$, die Unterstützung des Sprachprozesses durch den Studierenden ( $M e d{ }_{P D+I K K}=6$ ), $p=.37$ sowie die Tatsache, dass die Zehntklässler:innen im Drama Lab nicht durch die reguläre Klassenlehrerin unterrichtet wurden (Med $P D=3.4$; Med $\left.I_{K K}=3.2\right), p=.73$, keine statistisch bedeutsame Wirkung auf die Lernenden gehabt zu haben.

Die Sichtung der studentischen Planungsentwürfe ergab außerdem, dass die beiden unabhängigen Variablen, die studentischen IKK und PD-Lernangebote, tatsächlich zwei unterschiedliche methodische Schwerpunkte besitzen und damit konzeptuell durchaus als Einzelinterventionen trennbar sind. Unter insgesamt 27 IKK-Planungsentwürfen fanden sich jedoch lediglich sechs, bei denen Studierende zufälligerweise auf Rollenspiele zur Förderung von IKK setzten.

\subsection{Qualitative Ergebnisse}

Entlang der Forschungsfragen (FF) 1 bis 3 sollen nachfolgend die aus zwei Gruppendiskussionen von Drama Lab-Teilnehmenden gewonnenen Erkenntnisse berichtet werden:

\footnotetext{
${ }^{20}$ Da nicht ausgeschlossen werden konnte, dass die Studierenden für sowohl die IKK- als auch PD-Lernangebote auf Dramapädagogik zurückgreifen würden, wurde zunächst ein zweiseitiger Test durchgeführt, ohne die Annahme, dass die Lernenden ihre Sprechkompetenz bei PD-Angeboten positiver wahrnehmen würden.
} 
Klempin \& Bosse: Englischlernende bearbeiten interkulturelle und dramapädagogische Sprechaktivitäten

FF1: Wie schätzen Englischlehramtsstudierende den Mehrwert von IKK und PD-Lernangeboten ein?

\subsubsection{Studentisch eingeschätzter Mehrwert von Performativer Didaktik (PD)}

PD wird von den Englischlehramtsstudierenden als eine Möglichkeit zur Gestaltung offeneren Unterrichts unabhängig vom Kompetenzziel, dem Schul- oder Milieusetting dargestellt (BR11FR). Außerdem sei aus Studierendensicht PD in allen Klassen umsetzbar, selbst, wenn bestimmte Gegebenheiten, wie bspw. räumliche Beschränkungen, dessen Umsetzung erschweren. Komplementär dazu passen auch Aussagen, dass PD durch enorme Versatilität und Dynamik besteche (LU23CA; SA16BE). Außerdem wird von großem Enthusiasmus und enormer Begeisterung auf Seiten von sowohl Studierenden als auch Schüler:innen während der Begleitung und Durchführung der dramapädagogischen Spracherwerbsprozesse berichtet (BR11FR). Performatives sprachliches Handeln könne es Englischlehrpersonen zudem ermöglichen, Sprachenlernende aus ihrer Komfortzone hervorzulocken. PD-Unterricht weiche außerdem deutlich von einem transmissiven Lehren, d.h. einem eher konventionellen Lehrenden zentrierten Englischunterricht, ab und könne Englischlernende aufgrund der dadurch entstehenden Frei- und Aushandlungsräume besser zum sprachlichen Lernen anregen (LU23CA). Eng daran gekoppelt sei für die befragten Studierenden das PD-Potential, die Zentrierung von Unterricht auf die Lehrperson aufzulösen (LU23CA). Für manche ergab sich durch die Erprobung von PD-Aktivitäten im Rahmen des Drama Labs die Möglichkeit, den eigenen Aktivitätenentwurf und dessen Umsetzung aus der Lernendensicht heraus kritisch in den Blick zu nehmen. Im folgenden Zitat schildert der Studierende einen Perspektivwechsel, ein aktives Mitfühlen mit den Lernenden und ein kritisches Reflektieren der eigenen Beschulungsbiographie und der selbst konzipierten Dramaaktivität:

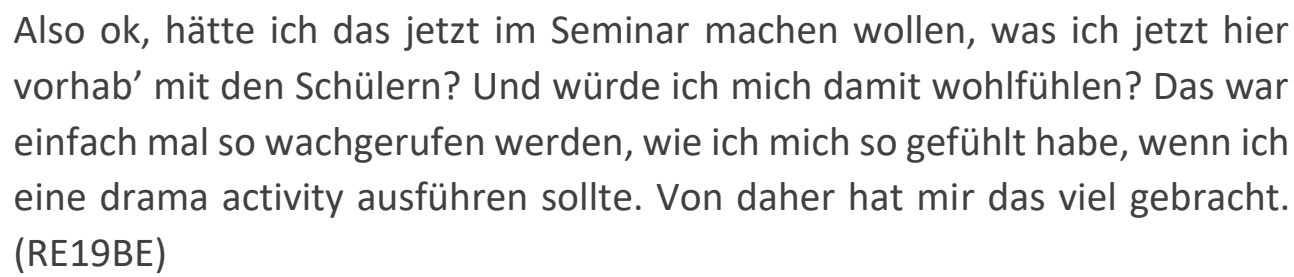

Performativ orientierter Unterricht ermögliche überdies eine völlig andersartige und „extremely nice“ (LU23CA) Form der Lernendeninteraktion. Ein weiterer Mehrwert von PD bestehe darin, dadurch kooperative Lernprozesse effektiver anbahnen und Entscheidungsfreiräume im Spracherwerbsprozess, beispielsweise durch eigenständige Rollenaushandlung und -arbeit, eröffnen zu können (SA16BE). 
Klempin \& Bosse: Englischlernende bearbeiten interkulturelle und dramapädagogische Sprechaktivitäten

\subsubsection{Studentisch eingeschätzter Mehrwert von Interkultureller Kommunikativer Kompetenz} (IKK)

Auch die IKK-Theoriebestände wurden von den vier Englischlehramtsstudierenden ${ }^{21}$ zunächst durchweg positiv bewertet. Davon zeugen die vielen positiven Horizonte ${ }^{22}$, die sich jedoch nicht auf den Mehrwert dieses Theoriegegenstandes für die L2 Lernenden beziehen, sondern auf die Rezeption durch die Lehramtsstudierenden (AN-08-MO: „wahnsinnig interessant" und "sehr bereichernd"; JN-12-BE: „sehr gut verständlich und hilfreich auch"). Die Studierenden heben in ihren Aussagen insbesondere die persönliche und/oder berufliche Relevanz von IKK für die Schulpraxis des Englischunterrichts hervor (JU-25-LI: „wirklich enorm wichtig und sehr sehr brauchbar"). Herausgehoben aus der Fülle der im Drama Lab aufgegriffen IKK-Theorien (u.a. Bach, 2007; Bredella, 2001; Freitag-Hild, 2018a/b; Küppers, 2015; Schmenk, 2010; Tomalin \& Stempleski, 1993; ) wird von den Studierenden das Byram-Modell (1997, S. 34ff.) mit seinen fünf "savoirs”. ${ }^{23}$ Dieses Modell habe den Studierenden Möglichkeiten zur Planung kompetenzorientierter IKK-Aktivitäten für den Schüler:innenbesuch eröffnet und Wege aufgezeigt, wie man zielgerichtet interkulturelle kommunikative Lernprozesse im Englischunterricht planen und anbahnen könne (AN-08-MO, JU-25-LI). An den Studierendenaussagen wird eine recht oberflächliche Auseinandersetzung mit der Modellierung interkultureller kommunikativer Kompetenz durch Byram evident. Das IKKModell von Byram wird von den Teilnehmenden an keiner Stelle näher beschrieben oder ausgeführt, es wird fast schon tropenhaft und mit großer Selbstverständlichkeit als totum pro parte, ohne jegliche theoretisch-begriffliche Explikation, verwendet. ${ }^{24}$ Byrams Modell und dessen Repräsentation im Berliner Rahmenlehrplan für moderne Sprachen (SenVerwBJW, 2014 , S. 9) habe den Studierenden ebenso zu Bewusstsein geführt, dass es bei der Planung und Umsetzung von Englischunterricht nicht ausschließlich darum ginge, in einer Unterrichtsstunde alle Facetten von IKK bei den Lernenden anbahnen zu müssen. Stattdessen

\footnotetext{
${ }^{21}$ Der Interviewer dieser Gruppe hat, entgegen vorheriger Absprache, die Studierenden nicht frei antworten lassen, sondern stattdessen die Anzahl der Redebeiträge von Anfang durch die Aussage "Drei, vier Leute können was dazu sagen" beschnitten. Die geringe Anzahl der Redebeiträge zu diesem Impuls kann daher vor dem Hintergrund betrachtet werden, wie hier der Impuls umformuliert und explizit vom Leitfaden abgewichen wurde und zwar dergestalt, dass sich dadurch keine natürlich-unbeeinflusste Diskursbewegung zu diesem Impuls entfalten konnte.

22 „Positiver Horizont“ ist eine Begrifflichkeit, die Bohnsack im Zuge der dokumentarischen Methode vorstellt.

${ }^{23}$ Byram (1997) beschreibt fünf interkulturelle Kompetenzdimensionen: 1. „savoir” als das soziokulturelle Wissen über die Eigen- und Fremdkultur, 2. „savoir être” als die für einen intercultural speaker (Byram, 1997, S. 32ff.) erforderlichen Einstellungen (u.a. Toleranz und Geistesoffenheit), 3. „savoir s'engager" als Fähigkeit zur kritischen Beurteilung der Eigen- und Fremdkultur, 4. „savoir comprendre” als Fertigkeiten des Interpretierens kommunikativer Szenarien von Sprechern aus unterschiedlichen Kulturkreisen, 5. „savoir apprendre/faire” als Interesse und Neugierde an der Exploration anderer Kulturen. Im Modell von Byram (1997) fehlt es noch an einer kinästhetischen Dimension, die später von Kessler (2008) ergänzt wurde.

24 An dieser Stelle bewegt sich die Interpretation vom immanenten, dem wörtlichen Sinn der Studierendenaussagen und bewegt sich in Richtung der reflektierenden Interpretation, die auf die Herausarbeitung kollektiver Orientierungsmuster der beiden Gruppen abzielt (Bohnsack 2014, S. 374-375).
} 
Klempin \& Bosse: Englischlernende bearbeiten interkulturelle und dramapädagogische Sprechaktivitäten

haben die angehenden Englischlehrenden im Drama Lab am Beispiel von IKK selbst erfahren können, dass die zielsprachliche Kompetenz mit einer gewissen Progression und zielgerichtetsystematisch aufgebaut werden könne (JU-25-LI). Neben der IKK-Modellierung durch Byram (1997) seien insbesondere solche Seminarlektüren bedeutsam gewesen, die „hilfreiche” Praxisbeispiele beinhaltet haben (JN-12-BE, u.a. Bachmann, Gerhold \& Wessling, 1996; Bolten, 1993; Bredella, 1997; Brunsmeier, 2016; Häussermann \& Piepho, 1996; Massler \& Storz, 2012; Surkamp, 2001; vgl. auch Beschreibung des Drama Lab-Konzeptes in Klempin, 2020). Neben den savoirs aus Byrams IKK-Modell wird ebenfalls betont, dass das Konzept des „critical incident[s]” (Bertallo et al., 2004, S. 26) sowohl "großartig” als auch „wirklich enorm wichtig“ und "sehr sehr brauchbar" für die Praxis der Planung und Durchführung eines kompetenzorientierten Englischunterrichts gewesen sei (JU-25-LI). ${ }^{25}$

\subsubsection{Fazit und Folgerungen zum studentisch eingeschätzten Mehrwert von PD und IKK}

Zusammenfassend sind die studentischen Relevanzurteile zum IKK-Theoriebaustein für das Lehren und Lernen einer Fremdsprache durch eine homologe repetitiv-varianzlose, fast schon gleichförmige Semantik gekennzeichnet (vgl. AN-08-MO und JU-25-LI: vier Mal Verwendung des Adjektivs „hilfreich”). Sie beurteilen den Mehrwert zwar einstimmig positiv, berichten jedoch an keiner Stelle ihre eigenen, die Erfahrungen ihrer Kommiliton:innen oder gar der Lernenden in der Begegnung mit diesen Theorien. Die IKK-Gruppe gerät eben nicht in ein erfahrungsbasiertes Erzählen wie es für die Reflexion des Mehrwerts von PD durch die PDGruppe herausgearbeitet werden konnte. ${ }^{26}$ Die IKK-Theorien werden indes vorrangig vor dem Hintergrund ihres Nutzens für die Planung eines kompetenzorientierten Englischunterrichts reflektiert. Die außergewöhnlichen Potentiale von IKK-Aktivitäten für die L2-Lernenden

\footnotetext{
${ }^{25}$ Die Englischlehramtsstudierenden lernten das Konzept des critical incidents im Drama Lab unter folgender Definition kennen (Küppers, 2015, S. 156): „anecdotes and real-life events in which misunderstandings occur which irritate, disturb, or even terminate communication due to differing cultural, social, or gender-based patterns of thinking or action of the speakers involved in the event". Gemäß dieser Definition waren die Studierenden dazu angehalten, eine ähnliche Kommunikationshürde zwischen zwei Sprechern aus einem von innen rezipierten Kapitel von Julian Barnes' „The History of the World in 10 1 2 Chapters” herauszuarbeiten und anschließend in eine IKK-Aktivität mit Fokus auf eine der vorgestellten savoirs zu überführen. Diese/r Studierende berichtet nun, dass die Identifikation eines ebensolchen critical incidents, eines fiktiven Kommunikationsereignisses, in einem rezipierten Kapitel aus „The History of the World in $101 / 2$ Chapters" ihn/sie unterstützt habe, auf Lektüregrundlage eine interkulturelle Kommunikationsaufgabe mit dem Ziel, eine der vier saviors bei den Englischlernenden zu entwickeln, zu konzipieren. Das Konzept des critical incidents stellte im Seminar für die Lehramtsstudierenden womöglich genau jene Brücke zwischen Literatur- Kulturwissenschaft und Englischdidaktik her und vermochte dadurch zwischen literarischer („,The History of the World in 101 1/2 Chapters") und theoretischer Lektüre (z.B. Byram, 1997; Surkamp, 2001), den Englischlernenden (Zehntklässler:innen eines Berliner Gymnasiums) und interkultureller kommunikativer Englischkompetenz (z.B. soziokulturelles Wissen und/oder interkulturelle Haltungen) zu vermitteln.

${ }^{26}$ Die Interpretation der Gruppendiskussion bewegt sich genau an dieser Stelle weg vom immanenten und hin zum dokumentarischen Sinngehalt, sprich, weg vom Wörtlichen (Was wird von den Studierenden gesagt?) hin zur Gestaltung des Diskurses durch die Studierendenbeiträge (Wie wird etwas gesagt?). Weitere reflektierende und komparative Interpretationen zur Diskursorganisation folgen in einem Anschlussbeitrag (Publikation in Vorbereitung).
} 
Klempin \& Bosse: Englischlernende bearbeiten interkulturelle und dramapädagogische Sprechaktivitäten

werden hingegen nicht kommentiert. Dies steht in Kontrast zu den studentischen Bewertungen der PD-Theorien, die mit enormem Detailreichtum, mit einer gewissen „metaphorische[n] Dichte” (Bohnsack, 2014, S. 34), in ihren vielfältigen Nutzungsspielräumen für den modernen Englischunterricht ausgewiesen werden. Zu manchen der genannten PDPotentiale zählen dessen Nutzungsvielfalt, Dynamik, starke Orientierung an den Bedürfnissen und Interessen sowie dessen Motivierungspotential für die L2-Lernenden. Im Gegenzug wird deutlich, dass die IKK-Theoriebestände durch Byrams theoretische Fundierung und die daraus entwickelten unterrichtspraktischen Handreichungen (u.a. Bachmann, Gerhold \& Wessling, 1996; Brunsmeier, 2016; Massler \& Storz, 2012; Surkamp, 2001) zur Gestaltung eines theoriebasierten und kompetenzorientierten Englischunterrichts durch die Studierenden deutlich zugänglicher sind und in der Folge für die Entwicklung eigener Aufgabenformate nutzbar waren. Maßgeblich dazu beigetragen hat scheinbar das IKK-Vier-SubdimensionenModell von Byram, dessen Aufgriff im Rahmenlehrplan für moderne Sprachen (SenVerwBJW, 2014, S. 9) und das von Bertallo et al. (2004, S. 26) eingeführte critical incident-Konzept. Letzteres ließ sich nach Studierendenaussagen relativ passgenau auf die Lektüre des literarischen Werks von Julian Barnes (2005) übertragen. Es erscheint auf Basis der ersten Analysen des empirischen Materials, der Studierendenwahrnehmungen, so, als wäre der PDim Vergleich zum IKK-Theoriediskurs weniger für den Fremdsprachenunterricht erschlossen und daher auch weniger anschlussfähig für die Ausbildung angehender Englischlehrer:innen. Zeitgleich besticht der PD-Ansatz durch seine vielfältigen Potentiale für den schulischen Erwerb des Englischen, wohingegen IKK-Theorien deutlich vor dem Hintergrund ihres Nutzens für die angehenden Englischlehrpersonen beschrieben werden.

Aus der unterschiedlichen kommunikativen Bearbeitung von IKK- und PD-Relevanz durch die Lehramtsstudierenden lassen sich möglicherweise erste (vorsichtige) Folgerungen ableiten:

- Der PD-Ansatz scheint für eine kompetenzorientierte Planung und Umsetzung von Englischunterricht noch nicht deutlich genug konturiert. PD besitzt in der Wahrnehmung von Lehramtsstudierenden, die diesen Ansatz selbst mit Zehntklässler:innen erprobt haben, allerdings enorme Vorzüge für die Planung eines an den Schüler:innen ausgerichteten, motivierenden und kognitiv aktivierenden Englischunterrichts.

- Im Gegensatz dazu existiert für den IKK-Theoriediskurs offenbar eine Ausschärfung des zugrundeliegenden Konstrukts. Ein Beispiel hierfür ist das Byram-Modell (1997), welches IKK in verschiedenen Kompetenzfacetten (sog. "savoirs“) modelliert und damit sowohl Möglichkeiten zur Planung und Realisierung kompetenzorientierten interkulturellen Englischunterrichts als auch zur psychometrischen Erfassung von IKK bzw. IKK-Überzeugungen verschiedener Lehr-Lern-Akteure eröffnet. 
Klempin \& Bosse: Englischlernende bearbeiten interkulturelle und dramapädagogische Sprechaktivitäten

FF2: Welche Hürden nehmen die Englischlehramtsstudierenden für den IKK- und PDLernprozess wahr?

Entsprechend studentischer Erfahrungen aus dem Drama Lab könnten performative Verfahren nur dann wirken, wenn zuvor zwischen Lehrendem und Lernenden die dafür notwendigen Voraussetzungen geschaffen wurden. Dazu zähle ein vertrauensvolles, geschütztes und wertschätzendes Umfeld, in dem dann handlungsorientiert die Zielsprache erkundet werden könne. Zu diesem Zweck könne der eigentlichen PD-Aktivität beispielsweise eine Art ,Eisbrecher' oder ,Aufwärmung' vorausgehen. Die hier befragten Studierenden berichten, dass insbesondere den PD-Aktivitäten vorausgehende interkulturell kommunikative Lernangebote den Studierenden und Lernenden Möglichkeiten boten, sich besser kennenzulernen. Nach Ansicht der befragten Studierenden schufen die IKK-Aktivitäten damit dasjenige Arbeitsklima, unter dem der dramapädagogische Spracherwerb stattfinden konnte. Denn erstaunlicherweise bargen die PD-Aktivitäten nicht nur ein enthemmendes Potential (vgl. auch FF1), sondern waren für manche studentische Lehrende wie auch Lernende eine verunsichernde Erfahrung (BR11FR). Als weitere Hürde für einen erfolgreichen dramapädagogischen Lernprozess identifizierten die Befragten hierarchische Differenzen. In vielen Klassenzimmern besteht noch immer eine starke Machtantinomie zwischen Lernenden und Lehrendem (Helsper, 2004). Für ein möglichst angstfreies Erfahren und Erproben von Sprache durch dramapädagogische Verfahren ist jedoch ein möglichst hierarchiearmer und wertneutraler Erfahrungsraum erforderlich. Nach Ansicht der befragten Studierenden könne Vertrauen aufgebaut und können Hierarchien abgebaut werden, wenn der PD- beispielsweise eine IKK-Aktivität vorausginge oder wenn, wie im Drama Lab, die Studierenden selbst noch schulpraktisch unerfahren, relativ jung und demnach näher an der Lebenswelt der Zehntklässler:innen seien (FU19BE; AN10HA).

Während die Lehramtsstudierenden bei der Reflexion der PD-Aktivitäten nicht nur die Eigen-, sondern auch die Lernendenperspektive einnahmen, zeigt sich im Vergleich dazu, dass die IKKGruppe erneut (vgl. auch FF1) ausschließlich aus der Eigenperspektive auf die im Seminar kennengelernten IKK-Theoriebestände blicken. Hürden für den Englischlehrenden oder die lernenden werden nicht konkretisiert. Es findet einzig Erwähnung, dass ausgeprägte sprachliche, soziale, inhaltliche, aber auch methodische Fertigkeiten der L2-Lernenden für eine erfolgreiche Spracharbeit der Englischlernenden im Zuge von IKK-Aufgaben von Nöten seien (Bach, 2007, S. 196).

FF3: Wie schätzen die Englischlehramtsstudierenden die Lernatmosphäre während der IKKund PD-Lernangebote ein? 
Klempin \& Bosse: Englischlernende bearbeiten interkulturelle und dramapädagogische Sprechaktivitäten

Konzeptionell sind dramapädagogische Aktivitäten für die Befragten zunächst mit der Befürchtung besetzt, dass diese bei Lernenden Sprech- und Performance-Ängste ${ }^{27}$ auslösen könnten (vgl. FF2). Die Erprobung eigener PD-Lernangebote im Zuge des Drama Labs konnte die Lehramtsstudierenden jedoch vom Gegenteil überzeugen. Die Lernatmosphäre zwischen der Schüler:innengruppe und den Studierenden während der PD-Aktivitäten wird emotional aufgeladen, fast schon überschwänglich und in großer metaphorischer Dichte mit zahlreichen Hyperbeln und Pleonasmen wie beispielsweise "super gut” ausgeschmückt (LU23CA). Die Atmosphäre zwischen Lernenden und angehenden Englischlehrenden sei deshalb so gut gewesen, weil sich die Schüler:innen bereits untereinander kannten, aber auch, weil diese die Studierenden bereits vom ersten Universitätsbesuch kannten. Die optimale Lernatmosphäre wird ebenfalls damit begründet, dass manche Lerngruppen eigene Räume für ihre Aktivitäten zur Verfügung stehen hatten („super super toll”) und es sich deshalb wie „VIP Unterricht” angefühlt habe (LU23CA). FU19BE nimmt an, dass sowohl die Universität als Lernort an sich („da laufen Studenten hin und her"), als auch der unkonventionelle Lernort innerhalb des Universitätsgebäudes („wir saßen im Flur”) zu der angenehmen Atmosphäre beigetragen haben könnten. Spekuliert wird darüber, ob aber nicht auch das Verhalten der studentischen Lehrenden einen gewissen Einfluss auf die „ganz entspannt[e]” (FU19BE) Lernumgebung gehabt haben könnte. Die äußerst angenehme Stimmung wird jedoch nicht alleinig auf studentisches Verhalten zurückgeführt, sondern vor allem auch auf das ungewöhnlich hohe Selbstvertrauen und das ausgeprägte Ausgangssprachvermögen der Schüler:innen. Selbst durch das ungewohnte Lernumfeld an der Universität schien dieses Selbstbewusstsein nicht zu irritieren sein. Die positive Resonanz der Schüler:innen war selbst für den/die befragte/n Studierende/n erwartungswidrig, vor allem, da diese/r deutlich werden lässt, er hätte in einer ähnlichen Situation als Lernender wohl deutlich weniger kooperativ und verständnisvoll gehandelt (FU19BE: „wär' ich auch so reingehauen”). SA16BE konstatiert weiter, dass insbesondere im Fremdsprachenunterricht ein antiautoritäres Auftreten der Lehrperson die Voraussetzung für den Aufbau einer solchen vertrauensvollen Schüler:innen-LehrendenBeziehung sei, unter der die Lernenden ohne jeglichen Erwartungs- oder Bewertungsdruck und unter größtmöglicher Entscheidungsfreiheit in der Fremdsprache kommunizieren können. Genau eine derartige kommunikationsförderliche, da hierarchie- und evaluationsfreie Beziehung sei während der PD-Aktivitäten für die Schüler:innen zustande gekommen und habe „super gute Ergebnisse” (SA16BE) hervorgebracht (vgl. auch FF1 und 2): „[Die] haben so viel gesprochen, weil die halt wussten, wir werden jetzt hier nicht benotet, das sind jetzt hier auch nur Studierende, die Lehrkräfte werden wollen und wir können jetzt hier sozusagen machen, was wir wollen". Gleichzeitig wird eingeräumt, dass es spezifische

\footnotetext{
27 Wir beziehen uns an dieser Stelle auf den Begriff der, performance', da es den Interviewten um die künstlerische Darbietung im Zuge der Dramaaktivität ging.
} 
Klempin \& Bosse: Englischlernende bearbeiten interkulturelle und dramapädagogische Sprechaktivitäten

Unterrichtssituationen gäbe, in denen ein gewisses Maß an Führung der Lernenden durch die Lehrperson sinnvoll sei, zum Beispiel im Kontext von Unterrichtsstörungen. Am Diskurs zur Lernatmosphäre während der PD-Aktivitäten ist bemerkenswert, dass erneut darüber reflektiert wurde, welchen Einfluss die Lehrperson auf Motivation und allgemeines Wohlbefinden hat.

Im Gegenzug war die Lernatmosphäre bei den IKK-Aktivitäten nach Aussage gleich mehrerer Studierender, „eigentlich ziemlich angespannt” (ME-28-BE; SY-06-BE: „Also bei meinen beiden Schülerinnen hat man den Druck auch sehr gespürt"). Als Gründe für diese eher ungünstige Stimmung werden die Videografieaufnahmen angeführt, die in einem der drei Räume zum ersten Schüler:innenbesuch stattfanden (ME-28-BE; SY-06-BE). Es wird aber auch vermutet, es könne an den Studierenden selbst gelegen haben (SY-06-BE: „Ich weiß nicht, ob es an den Kameras lag oder an mir"). Die Zehntklässler:innen seien insbesondere durch die Anwesenheit der Kameras sehr unter Druck geraten, hätten sich in der Folge „nicht frei entfalten” können. Weiterhin bringt ME-28-BE hervor, dass bei ihm/ihr die Peer-to-Peer-Unterstützung nicht sonderlich ausgeprägt gewesen sei („in meiner Gruppe war unter den Schülern leider auch nicht wirklich Hilfe da").

\section{Diskussion}

Hinsichtlich Wortschatzgröße und Satzkomplexität, Flüssigkeit, Konversationsfähigkeiten und Zusammenhängendes Sprechen beurteilten die befragten Englischlernenden ihre Fähigkeiten immer dann und mit statistischer Bedeutsamkeit besser, wenn der Befragung ein performatives Lernangebot vorausging. Damit stützt die vorliegende Studie die Erkenntnisse von Galante und Thomson (2017), Sirisrimangkorn und Suwanthep (2013) sowie Sirisrimangkorn (2018) (vgl. auch Kapitel 1.). Besonders die bessere Selbsteinschätzung der Konversationsfähigkeiten nach den PD-Aktivitäten könnte damit begründet werden, dass Mimik und Gestik in dramapädagogischen naturgemäß eine größere Rolle als in interkulturellen Sprachlernangeboten spielen. Auch wenn letztere Annahme nicht durch die qualitativen Daten dieser Studie untermauert wird, so existieren bereits eine Reihe an zuverlässigen Studien, die einem bewegungsgestützten Fremdsprachenerwerb diverse Vorteile zuschreiben (u.a. Dragović, 2019; Hille et al., 2010; Macedonia, 2013; Schilitz, 2018).

Um die differentiellen Wirkungen auf die wahrgenommene Sprechkompetenz der Englischlernenden für PD- und IKK-Lernangebote noch realistischer einschätzen zu können, lohnt ein Blick auf die berichteten Effektstärken. Im Schnitt konnten für alle Dimensionen der Sprechkompetenz (mit Ausnahme von Grammatischer Korrektheit und Zusammenhängendem Sprechen) mittelstarke Effekte ausgemacht werden. Deren Konfidenzintervalle zeugen allerdings von tatsächlichen Effektstärken zwischen kleinen bis großen (für Wortschatzgröße, 
Klempin \& Bosse: Englischlernende bearbeiten interkulturelle und dramapädagogische Sprechaktivitäten

Satzkomplexität, Konversationsfähigkeiten), kleinen bis mittelgroßen (für Zusammenhängendes Sprechen, Grammatische Korrektheit), beziehungsweise mittleren bis großen Effekten (für Flüssigkeit). Diese recht weit aufgespannten Konfidenzintervalle sind vermutlich auf die statistische Mortalitätsrate zurückzuführen, die zu einer Stichprobenreduktion um erhebliche neun Schüler:innen zur zweiten Erhebung beitrug.

Diese Studie löst die Forderung nach weiteren empirischen Nachweisen zum Mehrwert performativer Didaktik im Vergleich zu anderen Lehransätzen ein (vgl. Kapitel 1.). Die häufige Ansprache sprechmotivationaler Faktoren wie Spaß und Interesse liefert neue Hinweise dafür, dass sich die Schüler:innen durch dramapädagogische Kommunikationsangebote affektiv angesprochener fühlten. Durchweg positive Aussagen der Lehramtsstudierenden in den Gruppendiskussionen zur Lernatmosphäre während der PD-Aktivitäten erhärten diesen Befund. Diese neuen Ergebnisse sprechen damit erneut für einen Mehrwert performativer Didaktik hinsichtlich „affektive[r] und subjektive[r] Dimensionen“ (Schmenk, 2015, S. 42) und bestätigen zugleich Vorgängerstudien (u.a. Sirisrimangkorn \& Suwanthep, 2013; Sirisrimangkorn, 2018; Wirag \& Surkamp, 2020). Motivationsförderlich wirken performative Aktivitäten vermutlich deshalb, weil sie enthemmendes Potential besitzen. Dafür sprechen zumindest die Aussagen der Teilnehmenden. Motivierend sind sie aber womöglich auch deshalb, weil sie für viele Lernende schlichtweg neu und damit unerwartet sind und eine Abwechslung vom regulären (Sprach-)Unterricht darstellen (Stichwort: Novitätseffekt). Gleichzeitig wurde jedoch auch von einer scheinbar sprechhemmenden Wirkrichtung dramapädagogischer Aktivitäten berichtet. Als weitere schlüssige Erklärung für das höher beurteilte Interesse an und die erhöhte Motivation durch PD-Aktivitäten kann die empirisch gestützte Annahme angeführt werden, dass die für dramapädagogische Aufgabenformate typische Rollenübernahme für die Lernenden einen geschützteren Experimentierraum erzeugt. In der überwiegenden Zahl der IKK-Aktivitäten fehlte eine solche Rollenübernahme, bzw. das acting-in-role-Prinzip, welches in Galantes Studie (2018, S. 281) insbesondere schüchterne Schüler:innen zum Sprechen ermutigen und dadurch individuelle Sprechzeiten erhöhen konnte. IKK-Angebote boten zurückhaltenden Englischlernenden weniger Möglichkeiten zur Rollenübernahme, woraus geringe Sprechzeiten und dadurch negativere Selbsturteile der eigenen Sprechkompetenzen resultiert sein mögen. Sirisrimangkorns Studie (2018, S. 19) verwies zudem auf den Zusammenhang zwischen einer abschließenden Aufführung und einem motivationssteigernden Effekt der PD-Aktivität auf die Schüler:innen. Eine Mehrzahl der performativen Lernangebote des Drama Labs waren in der Tat kleinere Rollenspiele vor der Lerngruppe, die nicht bewertet wurden. Die Studierendenaussagen untermauern, dass insbesondere die Evaluationsfreiheit, Ungezwungenheit und geringe Hierarchieunterschiede zwischen studentischen Lehrenden und den Zehntklässler:innen 
Klempin \& Bosse: Englischlernende bearbeiten interkulturelle und dramapädagogische Sprechaktivitäten

während der PD-Aktivitäten zu einer grundlegend positiv-wertschätzenden und damit lernförderlichen Atmosphäre beigetragen haben.

Die vorliegende Studie hat auch verdeutlicht, dass Aufwärm- und Gewöhnungsphasen durch vorausgegangene PD- oder IKK-Lernangebote eine Rolle beim Abbau von Sprechhemmungen, zumindest für diese beiden Aktivitätentypen, spielen (zu PD-Aktivitäten: Elis, 2015, S. 94). Die Studierendenaussagen verweisen daneben darauf, dass insbesondere während der IKKAktivitäten geknüpfte Beziehungen zwischen Studierenden und Lernenden voraussetzungsreich für die PD-Aktivitäten gewesen sein mögen. Hinzu kamen bessere Raumbedingungen und eine grundsätzlich sehr positive Lernatmosphäre. Unabhängig vom Lernangebot (IKK oder PD) scheint eine fehlerfreundliche Haltung des/der (angehenden) Englischlehrenden während einer Sprechaktivität eine beträchtliche Rolle für die Sprechmotivation der L2-Lernenden zu spielen. Einer Einzelstudierendenaussage zufolge ermögliche das Drama Lab den Englischlehramtsstudierenden die Erfahrung, dass statt Fehlervermeidung und Akkuratheit (HE-11-SH; SA16BE) insbesondere Lernfreude (HE-11-SH) und das Erreichen eines Sprachflusses (SA16BE) im Zentrum der Englischvermittlung stehen solle und diese Selbsterkenntnis womöglich bereichernd für „alle Lehrkräfte“ sei (HE-11-SH).

Ebenfalls für beide Aktivitätentypen gleichermaßen bedeutsam scheint die Unterstützung des Sprachprozesses der Zehntklässler:innen durch die Studierenden zu sein. Wie auch beim Einfluss der Fehlerkultur kann hier darauf geschlossen werden, dass die Studierenden bei beiden Interventionen Hilfsmaßnahmen die gleiche Aufmerksamkeit widmeten. Der Tatsache, dass es sich bei der Lehrperson nicht um die reguläre Lehrperson handelte, wurde bei beiden Interventionen ein mittlerer Einfluss auf das Sprechen beigemessen. Es kann daher nicht gänzlich ausgeschlossen werden, dass die Selbsteinschätzungen der Lernenden bei ihrem regulären Englischlehrenden anders ausgefallen wären.

Die performative Didaktik sieht eine starke Lernendenaktivierung und -orientierung vor. Dies verbalisieren auch die Studierenden. Außerdem berichten sie von einer sowohl natürlicheren, kooperativeren sowie ungezwungeneren Interaktionsqualität (LU23CA; SA16BE) im Zuge dramapädagogischer Aktivitäten, zum einen zwischen den Lernenden, aber auch zwischen innen selbst und den Lernenden. Komplementär zu dieser Erkenntnis ergibt sich, dass die Schüler:innen den Einfluss der Peers auf die Lernatmosphäre nach den PD-Aktivitäten etwas besser bewerteten als nach den IKK-Aktivitäten. Allerdings kann hierbei nicht ausgeschlossen werden, dass sich die Schüler:innen beim zweiten Universitätsbesuch mit Mitschüler:innen an einer Station zusammenfanden, mit denen sie sich besonders gut verstanden.

Insgesamt weisen die Studienergebnisse in eine Richtung: Performative Didaktik könnte beim Einsatz im Englischunterricht durchaus sprechförderliche Wirkungen entfalten und dies 
Klempin \& Bosse: Englischlernende bearbeiten interkulturelle und dramapädagogische Sprechaktivitäten

insbesondere im Vergleich zu einem alternativen unterrichtlichen Kompetenzziel wie IKK, welches häufiger in die Nähe dramapädagogischer Verfahren gerückt wird (u.a. Belliveau \& Kim, 2013; Küppers, 2011). Im nachfolgenden Abschnitt soll nun auf die Studieneinschränkungen eingegangen werden, u.a., um die gewonnenen Erkenntnisse hinsichtlich ihrer Generalisierbarkeit, Zuverlässigkeit und Güte einordnen zu können.

\section{Limitationen}

Die berichteten Ergebnisse dieser Mixed Methods-Studie im Vertiefungsmodell (Mayring, 2001, S. 5) sollten unter den für sie geltenden Beschränkungen betrachtet werden. Zum einen handelt es sich beim Drama Lab nur um einen dem Englischunterricht ähnlichen Praxiskontext. Die ökologische Validität ist vermutlich noch gegeben (Bortz \& Döring, 2006, S. 727), aber dennoch liegt nahe, dass Unterschiede zu einer regulären Englischstunde an einer Schule hinsichtlich Komplexitätsreduktion für die Lehramtsstudierenden bestehen (vgl. Rehfeldt et al., 2018, z.B. geringere Gruppengröße, außerschulischer Lernort, usw.). Damit ist unklar, inwieweit die Befunde dieses Quasi-Experiments auf den Schulkontext übertragen werden können. Die Aussagen der Studierenden während der Gruppendiskussionen legen außerdem nahe, dass sich die beiden Durchführungs- und Erhebungskontexte zum Nachteil der Sprechförderlichkeit der IKK-Aktivitäten unterschieden haben mögen. Dies kann durchaus negativ auf die Durchführungsobjektivität eingewirkt haben (Bortz \& Döring, 2006, S. 195).

Beide im Drama Lab ausgeführten Aufgabenformate sind für die Lernenden neuartig gewesen. Aussagen der Lehrperson bestätigen, dass die Lernenden in ihrem Englischunterricht zuvor weder mit vergleichbaren IKK- noch PD-Aktivitäten in Berührung gekommen waren. Ein potentieller Novitätseffekt würde daher auf beide Erhebungen einwirken, zumal eine Gewöhnung an die Formate womöglich schon allein dadurch stattgefunden hat, dass die Lernenden vor der Datenerhebung mehrere Aktivitäten derselben Intervention ausgeführt hatten.

Die Ergebnisse dieser Studie beruhen auf Selbsteinschätzungen der Lernenden, wodurch sich die für solche Studien klassischen Beschränkungen ergeben (Braun et al., 2008, S. 32f.). Fremdurteile über die Sprechkompetenz der Schüler:innen wären daher für zukünftige Studien angeraten, auch um interindividuelle Unterschiede im Introspektionsgrad der Lernenden ausgleichen zu können (eine Einschränkung, die auch auf Boras (2020) Studie gewirkt haben könnte). Der Versuch, in dieser Studie externe Ratings auf Videografiedaten der Studierenden und Lernenden anzuwenden, konnte aufgrund einer Lieferverzögerung technischen Zubehörs leider nicht realisiert werden. Weitere Erhebungsiterationen gemäß dem ursprünglichen Solomon-Vier-Gruppen-Plan (Rost, 2013, S. 45; Solomon, 1949) könnten diesem Mangel jedoch (immer noch) begegnen. Aufgrund der gegenwärtigen Pandemie wird 
Klempin \& Bosse: Englischlernende bearbeiten interkulturelle und dramapädagogische Sprechaktivitäten

diese Studie jedoch (bis auf Weiteres) nicht in ihrem ursprünglichen Forschungsdesign weitergeführt werden können. Dies hat auch Auswirkungen auf das Gesamtdesign der Studie (vgl. Kapitel 2 und 3.): Die Unvollkommenheit des eingangs beschriebenen Solomon-VierGruppen-Designs hat nun zur Folge, dass es an einem Vortest zur Bestimmung einer Baseline der wahrgenommenen Sprechkompetenz der Lernenden ohne jegliche Behandlung (z.B. durch IKK oder PD-Aktivitäten) fehlt. Aus einem hoch komplexen und hochwertigen Forschungsdesign ist damit „Ein-Gruppen-Plan mit Vortest, Behandlung und Nachtest“ (Rost, 2013, S. 137) und damit ein „vor-experimentelles Design“ (Rost, 2013, S. 137) geworden. Die dafür geltenden Einschränkungen sind vielzählig: Beispielsweise hatte die erste IKKBehandlung vermutlich Einfluss auf die Selbsteinschätzungen der Schüler:innen zum zweiten Messzeitpunkt. Außerdem kann der Regelenglischunterricht zwischen erstem und zweitem Messzeitpunt auf die Sprechkompetenzwahrnehmung u.v.m. eingewirkt haben.

Die Ergebnisse dieser Studie stehen weiterhin vermutlich unter dem Einfluss, dass es im Fragebogen an solchen Kontrollfragen fehlte, die ein wahrheitsgemäßes und konzentriertes Ausfüllen des Fragebogens durch die Lernenden überprüfen (Rost, 2013, S. 154; 169). Der Verzicht auf derartige Kontrollfragen diente gleichzeitig aber dem Motivationserhalt der Lernenden während der Erhebung und verfolgte damit auch das Ziel, die Selbsturteile der Lernenden in ihrer Verlässlichkeit zu verbessern (Rost, 2013, S. 169). Für zukünftige (Replikations-)Studien wäre zudem angeraten, ein komplexeres statistisches Auswertungsverfahren zu wählen, wie beispielsweise eine ANCOVA, im Rahmen welcher Varianzen der wahrgenommenen Sprechkompetenz auch durch den Einfluss von Kovariablen (z.B. sprechmotivationale Variablen wie z.B. Interesse und Motivation) erklärt werden könnten.

8 Implikationen für die performative Didaktik, deren Beforschung und die Lehrkräftebildung

Aufgrund der dargelegten Studienlimitationen (vgl. Kapitel 7.) wären zukünftige Anschluss-, wenn nicht sogar exakte Replikationsstudien, idealerweise in einem ähnlichen LehrLernformat wie dem Drama Lab, wünschenswert. ${ }^{28}$ Die Installation von Vergleichsgruppen, die Durchführung von Prätests und mehrmethodischen Forschungsdesigns, die Einbindung von Kontrollfragen in das Messinstrument, die Verbesserung der Durchführungsobjektivität

\footnotetext{
28 Kontaktieren Sie Christiane Klempin bei Interesse an interdisziplinärem Austausch und an einer Forschungskooperation, die bspw. in einer Replikation bzw. Fortführung dieser Studie an Ihrem Universitätsstandort münden kann.
} 
Klempin \& Bosse: Englischlernende bearbeiten interkulturelle und dramapädagogische Sprechaktivitäten

sowie die Ergänzung der Selbst- durch zusätzliche Fremdeinschätzungen (z.B. durch externe Ratings) wären angeratene, da die Studienqualität steigernde Maßnahmen.

Zukünftige Forschungsbemühungen sollten sich vorzugsweise verstärkt auch den Bedingungen widmen, unter denen effektiver performativer Fremdsprachenerwerb stattfinden kann. Muss einer dramapädagogischen Aktivität bspw. eine Aufwärmphase vorausgehen und sollte sie durch eine Präsentationsphase abgeschlossen werden? Was passiert mit der allgemeinen, aber auch der sprachbezogenen Motivation und den Sprechängsten, wenn diese Phasen nicht vorhanden sind? All dies sind Forschungsfragen, denen noch intensiver nachgegangen werden könnte. Dies würde dann aber auch bedingen, dass der performativen Didaktik zunehmend aus der Perspektive eines Konstruktes begegnet wird, das man anhand trennscharfer Operationalisierungen zuverlässig (psychometrisch) messen kann (Bortz \& Döring, 2006, S. 53).

Eine andere Studie in diesem Zusammenhang verweist auf eine unzureichende Trennschärfe performativer Didaktik (Klempin et al., in Vorbereitung). Es zeigen sich darin diverse Verwandtschaften zu anderen, bereits explizierten, wenn auch ebenso schwer fassbaren Konstrukten wie der interkulturellen kommunikativen Kompetenz oder dem Communicative Language Teaching (CLT). Der ursprünglich dieser Studie zugrunde gelegte Solomon-VierGruppen-Plan verfolgt die Prämisse (vgl. Abschnitt 3.), diese drei Konzepte sauber voneinander zu trennen, um das dem performativen Lehren und Lernen Eigenständige herausarbeiten und ein zuverlässiges quantitatives Messinstrument entwickeln zu können.

Für den Forschungsdiskurs liefert diese Studie erste empirische Erkenntnisse zum Mehrwert performativer Lehr- und Lernverfahren für die von Lernenden wahrgenommene und selbst eingeschätzte Sprechkompetenz. Mit Ausnahme von Zusammenhängendem Sprechen und Grammatischer Korrektheit konnten alle anderen Dimensionen der Sprechkompetenz, zumindest in der Lernendenwahrnehmung, verbessert werden. Beides indiziert Trends und keinesfalls feststehende Tatsachen. Auch hier sollten noch weitere Studien folgen, um diese Tendenzen genauer zu beleuchten. Dass insbesondere grammatische Kompetenzwahrnehmungen nicht gesteigert werden konnten, verweist unseres Erachtens vermutlich auf ein Desiderat in der Ausbildung angehender Englischlehrender. Scheinbar konnten weder die PD- noch die IKK-Aktivitäten das Vertrauen der Englischlernenden in ihre grammatische Kompetenz stärken. Ein Blick auf die Aktivitätenentwürfe verdeutlicht auch, warum: Keine der geplanten Aktivitäten zielte auf ein grammatisches Phänomen ab. Das Kompetenzziel der meisten Planungsentwürfe lag hingegen vorrangig auf einem 
Klempin \& Bosse: Englischlernende bearbeiten interkulturelle und dramapädagogische Sprechaktivitäten

monologischen oder dialogischen, möglichst freien Gebrauch der englischen Sprache durch die Lernenden. ${ }^{29}$

\section{Bibliografie}

Bach, G. (2007). Interkulturelles Lernen. In J.-P. Timm (Hrsg.), Englisch lernen und lehren. Didaktik des Englischunterrichts (S. 319-327). Cornelsen.

Bachmann, S., Gerhold, S. \& Wessling, G. (1996). Aufgaben- und Übungstypologie zum interkulturellen Lernen mit Beispielen aus „Sichtwechsel-Neu“. Zielsprache Deutsch, 27(2), 7791.

Baldwin, P. (2004). With drama in mind: Real learning in imagined worlds network continuum education. New educational press.

Barnes, J. (2005). History of the world in 101/2 chapters (31. Aufl.). Picador.

Belliveau, G., \& Kim, W. (2013). Drama in L2 learning: A research synthesis. Scenario, 7(2), 6-26. https://doi.org/10.33178/scenario.7.2.2

Bertallo, A. (2004). Verwirrende Realitäten - Interkulturelle Kompetenz mit Critical Incidents trainieren. Pestolazzianum.

Bethmann, A., Buschle, C., Reiter, H. (2019). Kognitiv oder qualitativ? Pretest-Interviews in der Fragebogenentwicklung. In N. Menold \& T. Wolbring (Hrsg.), Qualitätssicherung sozialwissenschaftlicher Erhebungsinstrumente (S. 159-193). Springer VS. https://doi.org/10.1007/978-3-658-24517-7 6

Bohnsack, R. (2010). Rekonstruktive Sozialforschung. Einführung in qualitative Methoden. Budrich.

Bohnsack, R. (2014). Rekonstruktive Sozialforschung: Einführung in qualitative Methoden (8., durchgesehene Aufl). Budrich.

Bolten, J. (1993). Interaktiv-interkulturelles Fremdsprachenlernen. Zur Konzeption von Planspielen und Fallstudien im wirtschaftsbezogenen Fremdsprachenunterricht. In H. P. Kelz (Hrsg.), Internationale Kommunikation und Sprachkompetenz (S. 99-139). Beiträge zum Fachprogramm der Expolingua. Bonn

Bora, S.F. (2020). Curtain up! Enhancing L2 spontaneous and authentic speaking opportunities through play scripts and drama-based approaches. RELC Journal, 003368821988753. https://doi.org/10.1177/0033688219887536

\footnotetext{
${ }^{29}$ Für eine Förderung grammatischer Kompetenzen erfordert es ein performatives Grammatikerwerbsverfahren, wie es bereits existiert (Even, 2003). Mit Methoden aus dramatischen Kunstformen nähern sich die Lernenden bei Even (2003) der fremdsprachlichen Grammatik auf körperliche, ästhetische, soziale, emotionale und kognitive Weise an. Ein solches dramapädagogisch orientiertes Lehr- und Lernkonzept sorge dann - so die Fremdsprachendidaktikerin - „für intensive und nachhaltig wirksame Erfahrungen mit der fremden Sprache, ebne den Weg zur bewussten Einsicht in ihre Grammatikregeln und schaff[e] die Voraussetzungen für ganzheitliches und autonomieförderndes Grammatiklernen“ (Even, 2003, Klappentext).
} 
Klempin \& Bosse: Englischlernende bearbeiten interkulturelle und dramapädagogische Sprechaktivitäten

Bortz, J., \& Döring, N. (2006). Forschungsmethoden und Evaluation (4. Aufl.). Springer. https://doi.org/10.1007/978-3-540-33306-7

Bosenius, P. (2017). Assessing performative competence in German ELF-classrooms - The task of teachers and learners. Scenario, 11(2), 51-66. https://doi.org/10.33178/scenario.11.2.4

Braun, E., Gusy, B., Leidner, B. \& Hannover, B. (2008). Das Berliner Evaluationsinstrument für selbsteingeschätzte, studentische Kompetenzen (BEvaKomp) (Bd. 54, S. 30). GESIS - Leibniz-Institut für Sozialwissenschaften. https://doi.org/10.1026/0012-1924.54.1.30

Bredella, L. C. (1997). Interkulturelles Verstehen im Fremdsprachenunterricht: Do the Right Thing von Spike Lee. Anglistik und Englischunterricht, 61, 163-181.

Bredella, L. C. (2001). Zur Dialektik von Eigenem und Fremdem beim interkulturellen Verstehen. Der fremdsprachliche Unterricht. Englisch, 35(53), 10-14.

Brunsmeier, S. (2016). Raising intercultural awareness. Interkulturelle kommunikative Kompetenz: Eine Handlungsanleitung zur Aufgabenentwicklung. Grundschulmagazin Englisch, 14(3), 31-34.

Byram, M. (1997). Teaching and assessing intercultural communicative competence. Multilingual Matters.

Dragović, G. (2019). Fremdsprachenunterricht (ent-)dramatisieren. Eine empirische Untersuchung zur Effizienz des dramapädagogischen Ansatzes im schulischen DaF-Unterricht mit speziellem Fokus auf Grammatik. [Dissertation, Universität Freiburg].

Elis, F. (2015). Mit dramapädagogischen Methoden sprachliche und kommunikative Kompetenzen fördern. In W. Hallet \& C. Surkamp (Hrsg.), Dramendidaktik und Dramapädagogik im Fremdsprachenunterricht (S. 89-115). Wissenschaftlicher Verlag.

Even, S. (2003). Drama Grammatik: Dramapädagogische Ansätze für den Grammatikunterricht Deutsch als Fremdsprache. ludicium.

Falk, M., Hain, J., Marohn, F., Fischer, H. \& Michel, R. (2014). Statistik in Theorie und Praxis: Mit Anwendungen in R. Springer Verlag. https://doi.org/10.1007/978-3-642-55253-3

Freitag-Hild, B. (2018a). Teaching culture-intercultural Competence, transcultural learning, global education. In C. Surkamp \& B. Viebrock (Hrsg.), Teaching English as a foreign language: An introduction (S. 170-175). J.B. Metzler.

Freitag-Hild, B. (2018b). What is culture?. In C. Surkamp \& B. Viebrock (Hrsg.), Teaching English as a Foreign Language: An Introduction (S. 160-166). J.B. Metzler. https://doi.org/10.1007/978-3-47604480-8 9

Galante, A. \& Thomson, R. I. (2017): The effectiveness of drama as an instructional approach for the development of second language oral fluency, comprehensibility, and accentedness. TESOL Quarterly 51(1), 115-142. https://doi.org/10.1002/tesq.290

Gill, C. (2013): Enhancing the English-language oral skills of international students through drama. ELT 6(4), 29-41. https://doi.org/10.5539/elt.v6n4p29 
Klempin \& Bosse: Englischlernende bearbeiten interkulturelle und dramapädagogische Sprechaktivitäten

Grau, M., \& Würffel, N. (2003). Übungen zur interkulturellen Kommunikation. In: K.R. Bausch, H.

Christ \& H.-J. Krumm (Hrsg.), Handbuch Fremdsprachenunterricht (S. 312-314, 4. Aufl.). A. Francke Verlag.

Häussermann, U. \& Piepho, H. E. (1996). Aufgaben-Handbuch Deutsch als Fremdsprache: Abriss einer Aufgaben- und Übungstypologie. Iudicium.

Helsper, W. (2004). Antinomien, Widersprüche, Paradoxien: Lehrerarbeit - ein unmögliches Geschäft? Eine strukturtheoretisch-rekonstruktive Perspektive auf das Lehrerhandeln. In B. KochPriewe, F.-U. Kolbe \& J. Wildt (Hrsg.), Grundlagenforschung und mikrodidaktische Reformansätze zur Lehrerbildung (S. 49-98). Klinkhardt.

Hille, K., Vogt, K., Fritz, M. \& Sambanis, M. (2010). Szenisches Lernen im Fremdsprachenunterricht. Die Evaluation eines Schulversuchs. Diskurs Kindheits- und Jugendforschung, 5(3), 337-350.

Klempin, C. (2019). Reflexionskompetenz von Englischlehramtsstudierenden im Lehr-Lern-LaborSeminar: Eine Interventionsstudie zur Förderung und Messung. J.B. Metzler. https://www.springer.com/de/book/9783476051196

Klempin, C. (2020). Handreichung zur Lehr-Lern-Gelegenheit: Literarisches, interkulturelles und performatives Lehren lernen im Drama Lab. https://www.fu-berlin.de/sites/k2teach/news

Klempin, C., \& Wirag, A. (2020). Wie wirkt Dramapädagogik im Fremdsprachenunterricht? Ein Leitfaden zum Aufbau, zur Durchführung und Auswertung von Experimentalstudien. Scenario, 14(2), 96-115. https://doi.org/10.33178/scenario.14.2.6

Klempin, C., Lietz, A. \& Grubecki, D. (in Vorbereitung). Wie überzeugt sind Englischlehramtsstudierende von dramenpädagogischen Wissensinhalten und deren Relevanz für den modernen Englischunterricht?

Küppers, A. (2011). The DICE Consortium (2010), The DICE has been cast: Research findings and recommendations on educational theatre and drama (Vol. 1); Making a World of Difference. A DICE resource for practitioners on educational theatre and drama (Vol. 2). European Commission. Scenario, 5(1), 107-112. https://doi.org/10.33178/scenario.5.1.12

Küppers, A. (2015). Interkulturelle Kompetenzen, Dramapädagogik und Theaterwissenschaft. In W. Hallet \& C. Surkamp (Hrsg.), Dramendidaktik und Dramapädagogik im Fremdsprachenunterricht (S. 145-164). WVT Wissenschaftlicher Verlag.

Küppers, A. (2015). Interkulturelle Kompetenzen, Dramapädagogik und Theaterwissenschaft. In W. Hallet \& C. Surkamp (Hrsg.), Dramendidaktik und Dramapädagogik im Fremdsprachenunterricht (S. 145-164). WVT Wissenschaftlicher Verlag.

Macedonia, M. (2013). Mit Händen und Füßen. Gehirn und Geist, 1-2, 32-36.

Massler, U., \& Storz, E. (2012). Interkulturelles Lernen in einem E-Mail-Projekt. Email to Brazil. grundschulmagazin-englisch.de, 2, 9-13.

Mayring, P. (2001). Kombination und Integration qualitativer und quantitativer Analyse [31 Absätze]. Forum Qualitative Sozialforschung / Forum Qualitative Social Research, 2(1), Art. 6. http://nbnresolving.de/urn:nbn:de:0114-fqs010162 
Klempin \& Bosse: Englischlernende bearbeiten interkulturelle und dramapädagogische Sprechaktivitäten

Mayring, P. (2013). Combination and Integration of Qualitative and Quantitative Analysis. Forum:

Qualitative Social Research, 2(1), http://search.proquest.com/docview/867758593/?pqorigsite=primo.

Miladinović, D. (2019). Prinzipien eines performativen Fremdsprachenunterrichts. Eine Bestandsaufnahme. In S. Even, D. Miladinović \& B. Schmenk (Hrsg.), Lernbewegungen inszenieren: Performative Zugänge in der Sprach-, Literatur- und Kulturdidaktik. Festschrift für Manfred Schewe zum 65. Geburtstag (S. 7-22). Narr Francke Attempto.

Piazzoli, E. (2011): Process drama: the use of affective space to reduce language anxiety in the additional language learning classroom. RiDE: The Journal of Applied Theatre and Performance 16(4), 557-573. https://doi.org/10.1080/13569783.2011.617104

Piccardo, E. (2014). From communicative to action-oriented: A research pathways.

https://www.researchgate.net/publication/338177979 Piccardo E2014From Communicative to Action-oriented a Research Pathways

Rehfeldt, D., Seibert, D., Klempin, C., Mehrtens, T., Lücke, M., Sambanis, M., Köster, H. \& Nordmeier, V. (2018). Mythos Praxis um jeden Preis? Die Wurzeln und Modellierung des Lehr-Lern-Labors. die hochschullehre, 4, 90-114.

Rost, D. H. (2013). Interpretation und Bewertung pädagogisch-psychologischer Studien: Eine Einführung. Klinkhardt. https://doi.org/10.1007/s35834-013-0069-1

Schewe, M. (1993). Fremdsprache inszenieren: Zur Fundierung einer dramenpädagogischen Lehr- und Lernpraxis. Zentrum für pädagogische Berufspraxis. Zentrum für pädagogische Praxis Universität Oldenburg. https://cora.ucc.ie/handle/10468/561.

Schewe, M. (2015). Fokus Fachgeschichte: Die Dramapädagogik als Wegbereiterin einer performativen Fremdsprachendidaktik. In W. Hallet \& C. Surkamp (Hrsg.), Dramendidaktik und Dramapädagogik im Fremdsprachenunterricht (S. 20-36). WVT Wissenschaftlicher Verlag.

Schewe, M. (2017). Dramapädagogik. In C. Surkamp (Hrsg.), Metzler Lexikon Fremdsprachendidaktik (S. 49-51). Metzler.

Schilitz, J. (2018). Gestengestütztes Vokabellernen im Englischunterricht der gymnasialen Oberstufe. In H. Böttger \& M. Sambanis (Hrsg.), Focus on Evidence II - Netzwerke zwischen Fremdsprachendidaktik und Neurowissenschaften (S. 247-256). Narr Francke Attempto.

Schmenk, B. (2010). Intercultural speaker. In C. Surkamp (Hrsg.), Metzler Lexikon Fremdsprachendidaktik (S. 117-118). Metzler.

Schmenk, B. (2015). Dramapädagogik im Spiegel von Bildungsstandards, GeRS und Kompetenzdiskussionen. In: W. Hallet \& C. Surkamp (Hrsg.), Dramendidaktik und Dramapädagogik im Fremdsprachenunterricht (S. 37-50). WVT Wissenschaftlicher Verlag.

Senatsverwaltung für Bildung, Jugend und Wissenschaft (SenVerwBJW). (2014). Rahmenlehrplan für moderne Fremdsprachen, Teil C. Jahrgangstufen 1-10. Senatsverwaltung für Bildung, Jugend und Wissenschaft. 
Klempin \& Bosse: Englischlernende bearbeiten interkulturelle und dramapädagogische Sprechaktivitäten

Sirisrimangkorn, L. \& Suwanthep, J. (2013): The effects of integrated drama-based role play and student teams achievement division (STAD) on students' speaking skills and affective involvement. Scenario 7(2), 37-51. https://doi.org/10.33178/scenario.7.2.5

Sirisrimangkorn, L. (2018): The use of project-based learning focusing on drama to promote speaking skills of EFL learners. ALLS 9(6), 14-20. https://doi.org/10.7575/aiac.alls.v.9n.6p.14

Solomon, R. L. (1949). An extension of control group design. Psychological Bulletin, 46(2), 137-150. https://doi.org/10.1037/h0062958

Surkamp, C. (2001). Fremdverstehen durch short plays. Das Kurzdrama Survival in the South von M.A. Freeman. Der fremdsprachliche Unterricht Englisch, 5, 27-31.

Surkamp, C. (2017). Metzler Lexikon Fremdsprachendidaktik: Ansätze - Methoden - Grundbegriffe (2. Aufl.). J.B. Metzler. https://doi.org/10.1007/978-3-476-04474-7

Tomalin, B., \& Stempleski, S. (1993). Cultural awareness. Oxford: Oxford University

Trim, J., North, B. \& Coste, D. (2013). Gemeinsamer europäischer Referenzrahmen für Sprachen: lernen, lehren, beurteilen. Klett-Langenscheidt.

Wollschläger, D. (2017). Grundlagen der Datenanalyse mit R: Eine anwendungsorientierte Einführung (4. Aufl.) Springer Verlag. https://doi.org/10.1007/978-3-662-53670-4 